\title{
Familiarity Detection is an Intrinsic Property of Cortical Microcircuits with Bidirectional Synaptic Plasticity
}

\author{
(D)Xiaoyu Zhang, ${ }^{1}{ }^{\circ}$ Han Ju, ${ }^{2}{ }^{-1}$ Trevor B. Penney, ${ }^{3}$ and ${ }^{(}$Antonius M.J. VanDongen ${ }^{2}$
}

\section{DOI:http://dx.doi.org/10.1523/ENEURO.0361-16.2017}

${ }^{1}$ NUS Graduate School for Integrative Sciences and Engineering, National University of Singapore, 117456 Singapore,

${ }^{2}$ Program for Neuroscience and Behavioral Disorders, Duke-NUS Medical School, 169857 Singapore, and

${ }^{3}$ Department of Psychology, National University of Singapore, 117570 Singapore

\begin{abstract}
Humans instantly recognize a previously seen face as "familiar." To deepen our understanding of familiarity-novelty detection, we simulated biologically plausible neural network models of generic cortical microcircuits consisting of spiking neurons with random recurrent synaptic connections. NMDA receptor (NMDAR)-dependent synaptic plasticity was implemented to allow for unsupervised learning and bidirectional modifications. Network spiking activity evoked by sensory inputs consisting of face images altered synaptic efficacy, which resulted in the network responding more strongly to a previously seen face than a novel face. Network size determined how many faces could be accurately recognized as familiar. When the simulated model became sufficiently complex in structure, multiple familiarity traces could be retained in the same network by forming partially-overlapping subnetworks that differ slightly from each other, thereby resulting in a high storage capacity. Fisher's discriminant analysis was applied to identify critical neurons whose spiking activity predicted familiar input patterns. Intriguingly, as sensory exposure was prolonged, the selected critical neurons tended to appear at deeper layers of the network model, suggesting recruitment of additional circuits in the network for incremental information storage. We conclude that generic cortical microcircuits with bidirectional synaptic plasticity have an intrinsic ability to detect familiar inputs. This ability does not require a specialized wiring diagram or supervision and can therefore be expected to emerge naturally in developing cortical circuits.
\end{abstract}

Key words: familiarity; learning; NMDA receptor; plasticity; recognition memory

\section{Significance Statement}

Humans recognize familiar faces instantly. The cellular mechanisms underlying this recognition memory are still poorly understood. Simulations presented here demonstrate that bidirectional synaptic plasticity is sufficient to endow recurrent spiking neuronal network models with the ability to detect familiar sensory inputs through unsupervised learning. Network spiking activity evoked by a face image results in changes in synaptic connectivity and the formation of a unique strengthened subnetwork. Networks can recognize multiple previously seen faces with high accuracy by forming partially overlapping subnetworks. We therefore propose that familiarity detection is an intrinsic property of generic cortical microcircuits with bidirectional synaptic plasticity.

\section{Introduction}

Recognition memory refers to the ability to recognize previously experienced sensory inputs. Prior studies

Received December 6, 2016; accepted April 27, 2017; First published May 8, 2017.

The authors declare no competing financial interests.
(Nickerson, 1965; Shepard, 1967) found that immediately following a single exposure to 612 pictures, subjects could select the previously-seen picture in two-alternative 
recognition tests with $98 \%$ accuracy. Later, Standing (1973) reported that the number of pictures correctly recognized increases with the number of pictures presented (up to 10,000), suggesting a limitless capacity of recognition memory. These experimental observations can be accounted for by familiarity, a form of unsupervised learning. Familiarity and recollection are two major processes that underlie recognition memory (Yonelinas, 2002; Squire et al., 2007). While recollection demands accurate recall of the object's features, familiarity merely requires a signal indicating that the object has been previously encountered (Wixted, 2007). Evidence from functional imaging studies shows that cortical regions surrounding the hippocampus become active when a human subject senses a familiar input, whereas activation of the hippocampus is required for recollection (Hölscher et al., 2003; Diana et al., 2007).

While learning and memory have been extensively studied at the molecular/cellular (Wu et al., 2006; Baudry et al., 2015) and behavioral (Gale et al., 2014; Rapanelli et al., 2015) levels, it has been difficult to causally connect these two levels of desciption (Morgado-Bernal, 2011). Memories are believed to be encoded by and stored in a subset of neurons (the "engram"), which are connected by synapses whose weights were altered by the learning experience (Takeuchi et al., 2014). Given the current knowledge on how learning induces changes in synaptic efficacy through long-term potentiation (LTP; Bliss and Lomo, 1973), longterm depression (LTD; Ito and Kano, 1982; Massey and Bashir, 2007), and spike-timing-dependent plasticity (STDP; Gerstner et al., 1996; Markram et al., 1997; Bi and Poo, 1998), it is meaningful to simulate the synaptic changes in biologically plausible neural networks, to foster understanding from a systems perspective.

The liquid state machine (LSM) is a biologically-inspired spiking neural network model, which closely emulates the complexity of a generic cortical microcircuit. It is designed to perform biologically realistic real-time computing on time-varying inputs, providing an alternative to the widelyused attractor neural networks which require convergence to stable internal states (Maass et al., 2002). The construction of an LSM network, which involves generating random connections with random synaptic weights, is task independent. An important property of these networks is that sensory inputs are expanded into highdimensional feature space, allowing linear separation of complex properties (Buonomano and Maass, 2009). Temporal and spatial input information is transiently preserved

This work was supported by grants MOE2012-T2-1-039 from the Singapore Ministry of Education and OFIRG/0019/2016 from the National Medical Research Council (to A.M.J.V.) and by an award from the Singapore Ministry of Health and A*STAR, the Agency for Science, Technology and Research.

Correspondence should be addressed to Antonius M.J. VanDongen, Program for Neuroscience and Behavioral Disorders, Duke-NUS Medical School, Singapore 169857, E-mail: antonius.vandongen@duke-nus.edu.sg.

DOI:http://dx.doi.org/10.1523/ENEURO.0361-16.2017

Copyright (C) 2017 Zhang et al.

This is an open-access article distributed under the terms of the Creative Commons Attribution 4.0 International license, which permits unrestricted use, distribution and reproduction in any medium provided that the original work is properly attributed. in the form of fading memory, through multiple recurrent loops and short-term synaptic plasticity (depression and facilitation). We have introduced activity-dependent longterm synaptic plasticity into the network model by incorporating NMDA receptor (NMDAR) functionality (Shouval et al., 2002) in the excitatory synapses.

NMDARs are critically important for synaptic plasticitydependent learning. When post-synaptic depolarization coincides with glutamate and glycine binding, NMDARs open and allow $\mathrm{Ca}^{2+}$ influx (Blanke and VanDongen, 2009). Depending on the amount of $\mathrm{Ca}^{2+}$ influx, it will selectively activate phosphatases (low calcium influx) or the kinase CaMKII (high calcium influx), and trigger downstream signaling for synaptic depression or potentiation, respectively (Salter et al., 2009; Malleret et al., 2010; Luscher and Malenka, 2012), a theory known as the calcium control hypothesis (Lisman, 1989). LTP and LTD can be induced by tetanic synaptic inputs that regulate the $\mathrm{Ca}^{2+}$ influx. They are rate-based: low-frequency stimulation causes LTD, while a high-frequency tetanus induces LTP. STDP is another form of synaptic plasticity regulated by the temporal correlation of pre- and post-synaptic firing. By implementing back-propagating action potentials (BPAPs), NMDAR functionality can support STDP through calcium control (Waters et al., 2005; Paradiso and Wu, 2009). We have implemented both rate-based (LTP/LTD) and spike-timing-based (STDP) plasticity, by modeling NMDAR functionality in the excitatory synapses using the calcium control hypothesis.

The NMDAR-containing neural network is a model of generic cortical microcircuits with the capability of unsupervised learning. We have used it here to study how familiarity could develop in the cortex. On a large scale, brain regions are wired into relatively deterministic neural circuits (Coutlee and Huettel, 2012; Fornito et al., 2012), yet randomness and flexibility prevails within local cortical regions, with functional connections being optimized by activity-dependent changes (Sporns and Zwi, 2004; Fair et al., 2009). The main hypothesis underlying our simulations is that a sensory stimulus induces changes in synaptic weights in a neural microcircuit, altering the network response such that it can distinguish familiar from novel inputs.

\section{Materials and Methods}

\section{Neural network implementation}

Neural networks were simulated using MATLAB and the CSIM package (a neural Circuit SIMulator, RRID: SCR_014256, available at http://www.lsm.tugraz.at/csim/), as described previously (Natschläger et al., 2002; Ju et al., 2013). NMDAR-dependent synaptic plasticity was introduced into the excitatory synapses following the model proposed by Shouval et al. (2002). The neural networks consist of two parts: an input layer, and the network reservoir. Input neurons send spikes to the network reservoir via static spiking synapses, which have no plasticity. The network reservoir consists of leaky integrate-and-fire (LIF) neurons recurrently connected by NMDAR synapses. Seventy-five percent of the neurons are set as excitatory, the remaining being inhib- 
itory. Each LIF neuron is modeled by a linear differential equation:

$$
\tau_{m} \frac{\mathrm{dV}_{m}}{\mathrm{dt}}=-\left(\mathrm{V}_{m}-\mathrm{V}_{\text {resting }}\right)+\mathrm{R}_{m}\left(\mathrm{l}_{\text {syn }}+\mathrm{I}_{\text {inject }}+\mathrm{I}_{\text {noise }}\right),
$$

where the parameters are: membrane time constant $\tau_{\mathrm{m}}=$ $30 \mathrm{~ms}, V_{\text {resting }}=0 \mathrm{mV}$, membrane resistance $R_{\mathrm{m}}=1 \mathrm{M} \Omega$, input currents supplied by explicitly modeled synapses $I_{\text {syn }}$, steady background current $I_{\text {inject }}=13.5 \mathrm{nA}$ and for some simulations random noise $I_{\text {noise }}$ was added to the current. For the first time step in the simulation, the membrane potential $V_{m}$ was set to an initial random value between -1 and $1 \mathrm{mV}$. When $\mathrm{V}_{\mathrm{m}}$ increases to $15 \mathrm{mV}$ (the firing threshold), the neuron fires, and $V_{m}$ is reset to a random value between -1 and $1 \mathrm{mV}$ after an absolute refractory period of $3 \mathrm{~ms}$ for excitatory neurons and $2 \mathrm{~ms}$ for inhibitory neurons (Joshi, 2007).

In CSIM, the probability that two neurons are connected by a synapse is defined as:

$$
P(D)=C \cdot \exp \left(\frac{-D^{2}(a, b)}{\lambda^{2}}\right),
$$

where $D(a, b)$ stands for the Euclidean distance between the two neurons $a$ and $b . \lambda$ and $C$ are parameters used by CSIM that determine connectivity and synaptic strength, respectively. As $\lambda$ increases, both the connection probability and the average connection length will increase. The base value of $C$ depends on the type of connection: it is set to $0.3,0.2,0.4$, and 0.1 for EE, El IE, and II connections, where $E$ and I stand for excitatory and inhibitory neurons. The values are based on recordings from rodent cortical brain areas (Gupta et al., 2000). The actual value of $\mathrm{C}$ is modulated by a user-defined parameter, Cscale. Input layer neurons are all excitatory. Connections from input neurons to the network reservoir and within the network reservoir are randomly generated following the probability $P(D)$.

Once a connection is established, it is assigned an initial synaptic weight, indicating synaptic efficacy. Initial synaptic weights are drawn from the following gamma distribution:

$$
\begin{aligned}
& y=f(x \mid a, b)=\frac{1}{b^{a} \Gamma(a)} x^{a-1} e^{\frac{-x}{b}} \\
& a=\frac{1}{S H \_W^{2}}, b=W \cdot S H \_W^{2},
\end{aligned}
$$

where $\Gamma(\cdot)$ is the Gamma function. $S H \_W$ and $W$ are parameters used by CSIM. SH_W (default 0.7 ) positively correlates with the variance of the weight distribution and $W$ correlates with the mean of the distribution. The base value of $W$ is set to $3 \mathrm{e}^{-8}$ for $\mathrm{EE}, 6 \mathrm{e}^{-8}$ for $\mathrm{El},-1.9 \mathrm{e}^{-8}$ for IE and II. The actual value of $W$ is modulated by a user defined parameter, Wscale. The synaptic weight of an excitatory NMDAR synapse is subject to strengthening (upper boundary $=6.5 \mathrm{e}^{-8}$ ) or weakening (lower boundary $=1.0 \mathrm{e}^{-9}$ ) by plasticity. Synapses from the inhibitory neurons have negative weights, and do not possess plastic- ity. Synaptic weights of the static spiking synapses from input neurons are fixed.

In CSIM, a network is generated by placing neurons on a 3-D grid. The networks described in Figure $1 A$ had five layers with $10 \times 10$ neurons each (dimension, $10 \times 10 \times$ 5). Input neurons formed synapses one-to-one with the first layer of the network reservoir, with fixed synaptic weights of $2.7 \mathrm{e}^{-7}$. NMDAR synapses in the network reservoir were generated with $\lambda=2.0$ and Cscale $=1.0$. Initial weights followed the gamma distribution with SH_W $=0.25$ and Wscale $=0.5$.

Networks described in Figure $1 B$ consisted of five or six layers, with dimensions $20 \times 20 \times 5,50 \times 50 \times 5$, and $50 \times 50 \times 6$. In this case, input neurons formed synapses randomly with the network reservoir with Cscale $=0.04$, 0.004 , and 0.005 , respectively, and $\lambda$ was set to infinity in all cases to remove the limitation by distance. As a result, there was no topographical mapping of the input pattern. Input synaptic weights were still fixed but no longer uniform, following a gamma distribution (Wscale $=3, \mathrm{SH} \_\mathrm{W}=0.7$ in all cases) instead. As for NMDAR synapses in the network reservoir, $\lambda=4.0$ for the $20 \times 20 \times 5$ networks; $\lambda=3.0$ for $50 \times 50 \times 5$ and $50 \times 50 \times 6$ networks; Cscale $=1$ for all cases. The $\lambda$ values were chosen to make sure that each neuron formed $\sim 100$ synapses on average with others in the network. Initial weights of NMDAR synapses also followed a gamma distribution with Wscale $=0.9$ and SH_W $=0.25$. By setting Wscale to 0.9 , the initial weights were set to intermediate values, leaving enough room for future potentiation and depression. By setting $\mathrm{SH} \_\mathrm{W}$ to 0.25 for the network reservoir, we reduced the variation in the initial weights, thereby reducing any preimposed network circuitry.

\section{Synaptic plasticity implementation}

The NMDAR-dependent plasticity we implement follows the model by Shouval et al. (2002). Synaptic plasticity (LTP/LTD and STDP) depends critically on the amplitude and timing of postsynaptic EPSPs and BPAPs. BPAPs were not implemented in the original CSIM, while EPSPs were implemented using a single exponential decay function with a time constant of $3 \mathrm{~ms}$. We introduced BPAPs and changed both BPAPs and EPSPs to follow double-exponential decays, each with a fast and a slow component. Decay time constants were scaled from the suggested values (Shouval et al., 2002), with BPAP fast decay time constant $\tau_{f}^{b s}=1.2 \mathrm{~ms}$ and proportion $I_{f}^{\text {bs }}=$ 0.75 , slow decay time constant $\tau_{s}^{b s}=10 \mathrm{~ms}$ and proportion $l_{s}^{b s}=0.25$; EPSP fast decay time constant $\tau_{f}^{e p}=2 \mathrm{~ms}$, proportion $l_{f}^{e p}=0.5$, slow decay time constant $\tau_{s}^{e p}=20 \mathrm{~ms}$ and proportion $l_{f}^{e p}=0.5$.

$$
\begin{aligned}
& B P A P(t)=B P A P \_\max \cdot\left(l_{f}^{b s} e^{-t / \tau_{f}^{b s}}+l_{s}^{b s} e^{-t / \tau_{s}^{b s}}\right) \\
& E P S P(t)=E P S P \_m a x \cdot\left(l_{f}^{e p} e^{-t / \tau_{f}^{\rho p}}+l_{s}^{e p} e^{-t / \tau_{s}^{e p}}\right)
\end{aligned}
$$

Using a double-exponential decay ensures that the BPAP has a sharp peak with a thin tail and that the EPSP has a slower peak and more prominent tail, thereby preserving the difference in temporal signature between 
A

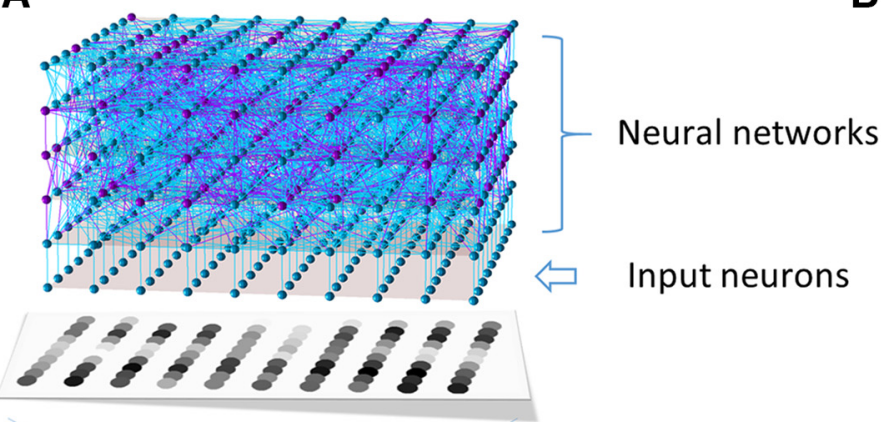

B

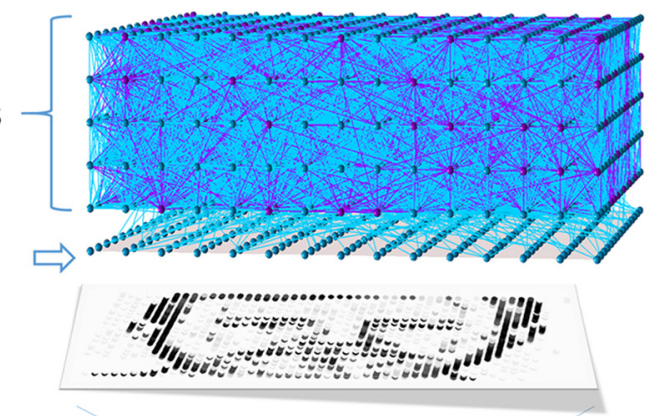

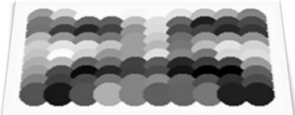

C



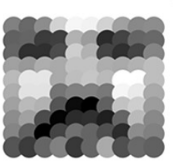

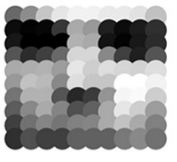

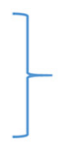

Face stimuli

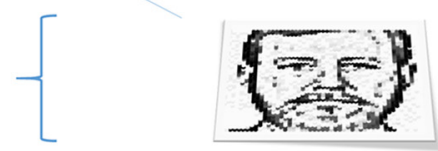

E

F

Figure 1. Network architecture and stimulus encoding. $\boldsymbol{A}$, Diagram illustrating a $10 \times 10 \times 5$ network with a $10 \times 10$ input layer, receiving a stimulus from a beard face image $(10 \times 10$ pixels). Neurons in the network reservoir are located at positions with integer coordinates in a 3-D space. The input layer is located 1 unit away from the network reservoir. Input neurons form synapses one-to-one with the first layer of the network reservoir. Each input neuron receives a spike train with a firing rate determined by the corresponding image pixel intensity value. $\boldsymbol{B}$, Diagram illustrating a $50 \times 50 \times 5$ network (only a portion of size $15 \times 15 \times 5$ is shown) with a $50 \times$ 50 input layer (only $15 \times 15$ portion is shown), receiving a stimulus from a human face image $(50 \times 50$ pixels). Input neurons form synapses with random neurons in the network reservoir. $C$, Examples of a $10 \times 10$-pixel beard face (top) and a $10 \times 10$-pixel no-beard face (bottom). $\boldsymbol{D}$, Examples of $20 \times 20$-pixel images of car fronts, dog faces and human faces. $\boldsymbol{E}$, Examples of $50 \times 50$-pixel human faces. $\boldsymbol{F}$, A sample beard stimulus which contains spike trains $(0-0.5 \mathrm{~s})$, followed by a silent interval $(0.5-1.0 \mathrm{~s})$. Only 50 channels of spike trains are shown for clarity.

EPSPs and BPAPs. This contrast ensures that the change in intracellular $\mathrm{Ca}^{2+}$ concentration induced by pre-post firing exceeds the $\left[\mathrm{Ca}^{2+}\right]$ change induced by post-pre firing, so that STDP will be induced properly (see Fig. 2 in Shouval et al., 2002).

Activity-induced EPSPs generate the driving force for calcium currents through the NMDAR:

$$
H(V)=\frac{-0.42\left(\mathrm{~V}-\mathrm{V}_{r}\right)}{\left(1+0.6 e^{-0.09 \mathrm{~V}} \cdot\left[\mathrm{Mg}^{2+}\right] / 3.57\right)}
$$

where $V=V_{\text {resting }}+$ EPSP + BPAP, and $V_{r}$ is the reversal membrane potential for calcium $(130 \mathrm{mV})$. The $\mathrm{Mg}^{2+}$ concentration is set to $1 \mathrm{mM}$. The driving force function was modified from the function suggested by Shouval et al. (2002) which did not take into consideration the effect of growing synaptic weights. In vitro recordings of ion currents through NMDARs (Pattillo et al., 1999) show a steeper curve, and based on this, we re-parameterized the calcium driving force (Fig. $2 A$ ).
The calcium current through NMDARs $\left(I_{\text {NMDA }}\right)$ is assumed to have this form:

$$
I_{N M D A}\left(t_{i}\right)=P_{0} G_{N M D A}\left[l_{f} \theta(t) e^{-t / \tau_{f}}+I_{s} \theta(t) e^{-t / \tau_{s}}\right] H(V)
$$

where $P_{O}$ is the probability of opening, $G$ is the conductance of the NMDAR channel, $l_{\mathrm{f}}$ and $l_{\mathrm{s}}$ are the fast and slow components of the NMDA currents, $\tau_{\mathrm{f}}$ and $\tau_{\mathrm{s}}$ are the time constants for the fast and slow components.

\section{Calcium control hypothesis}

The activity-dependent change in synaptic calcium concentration is modeled as a function of the NMDA current $I_{\text {NMDA }}$ :

$$
\frac{d C a(t)}{d t}=I_{N M D A}(t)-\left(1 / \tau_{C a}\right)[C a(t)]
$$

where $[\mathrm{Ca}(t)]$ is the calcium concentration at the synapse at time $t$ and $\tau_{C a}$ is the decay time constant, which is 
A

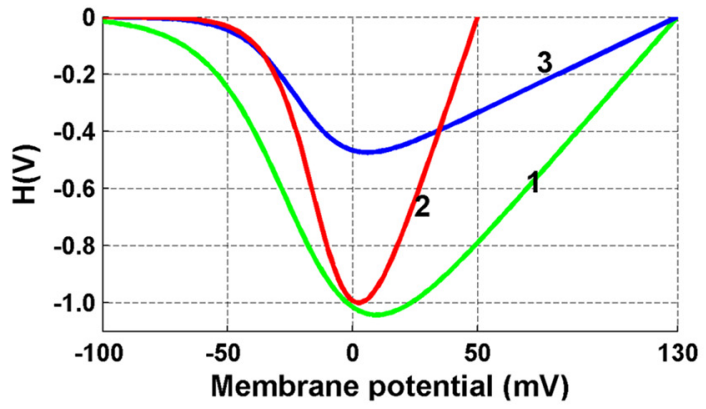

C

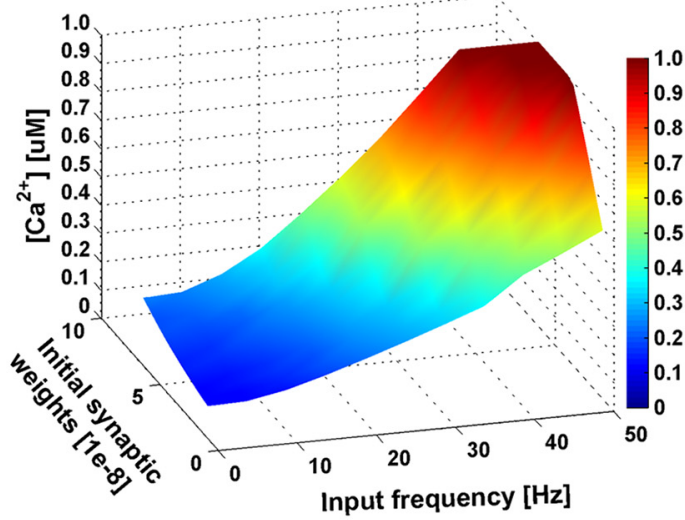

E
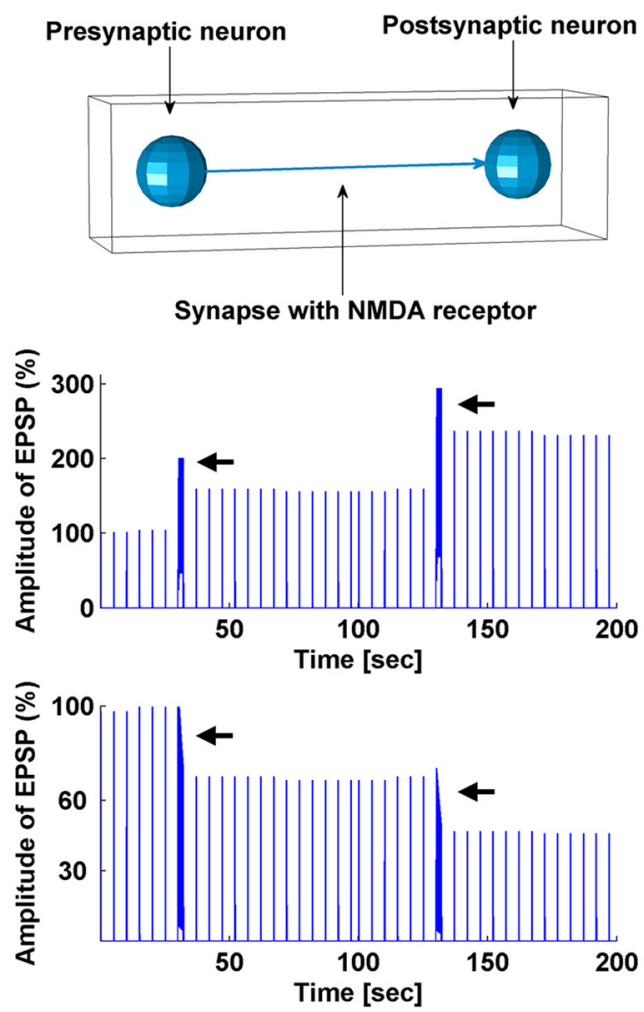

B

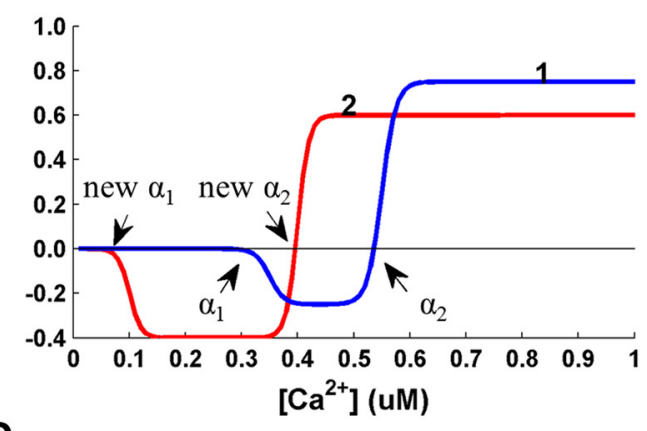

D

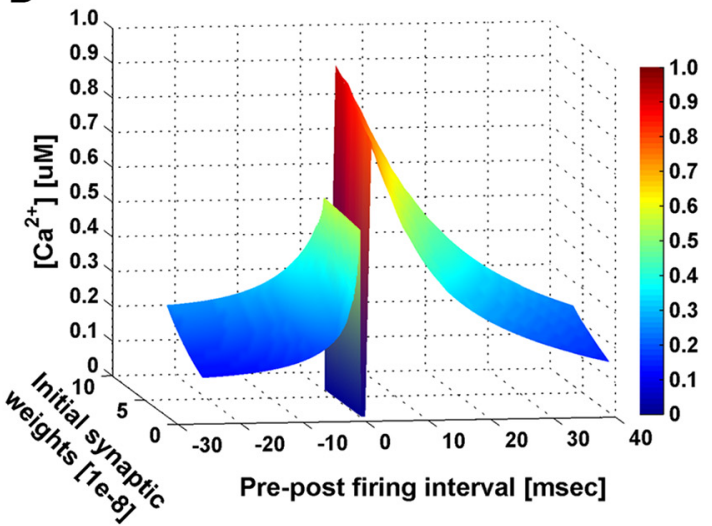

$\mathbf{F}$

Presynaptic neuron Postsynaptic neuron
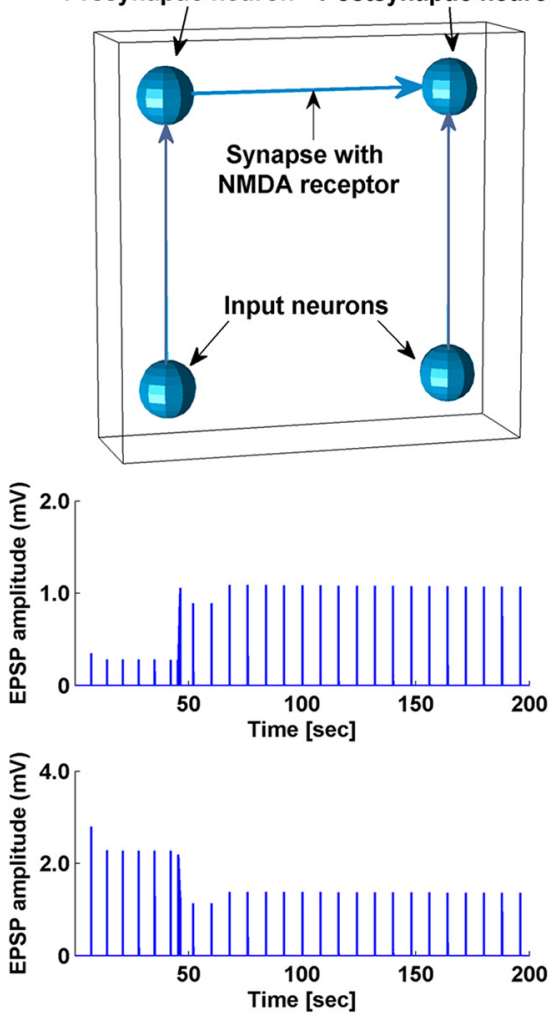

Figure 2. Parameter turning. $\boldsymbol{A}-\boldsymbol{D}$, Calcium control hypothesis. $A$, Curves illustrating the driving force function $\mathrm{H}(\mathrm{V})$ : (1) as proposed in (Shouval et al., 2002); (2) from in vitro recordings of ion currents through NMDARs (Pattillo et al., 1999); (3) re-parameterized in our model. Curve (3) is similar to (2) at negative membrane potentials. Curve (3) has the same equilibrium potential as (1) but is smaller in amplitude, reducing the impact of growing synaptic weights. $\boldsymbol{B}$, Models of the calcium control hypothesis $\Omega$ function: (1) as 


\section{continued}

proposed in (Shouval et al., 2002), (2) as used in our simulations. When real-time synaptic $\mathrm{Ca}^{2+}$ concentration ([Ca $\left.{ }^{2+}\right]$ ) exceeds the new $\alpha_{1}$ but not the new $\alpha_{2}$, synaptic weight is decreased; when real-time synaptic $\left[\mathrm{Ca}^{2+}\right]$ exceeds the new $\alpha_{2}$, synaptic weight is increased. $\boldsymbol{C}$, Real-time postsynaptic $\left[\mathrm{Ca}^{2+}\right]$ is plotted as a function of input frequency and initial synaptic weights. $\boldsymbol{D}$, Real-time postsynaptic $\left[\mathrm{Ca}^{2+}\right]$ is plotted as a function of pre-post firing intervals and initial synaptic weights. $\boldsymbol{E}$, Induction of LTP/LTD at individual synapses following high/low-frequency stimulation protocols. Top, Model setup. A presynaptic input neuron is connected to a postsynaptic neuron by an excitatory NMDAR synapse. The presynaptic input neuron generated spikes at a certain frequency, and EPSPs were recorded in the postsynaptic neuron. The postsynaptic neuron is prevented from firing action potentials. Middle, Simulation results with $50 \mathrm{~Hz}$ tetanus input on the model. Spike trains of $50 \mathrm{~Hz}$ were generated by the presynaptic input neuron, which caused long-lasting increases of the EPSP amplitude in the postsynaptic neuron. Arrows indicate when a $50 \mathrm{~Hz}$ tetanus $(2 \mathrm{~s})$ was applied. Bottom, Simulation results with $20 \mathrm{~Hz}$ tetanus input on the model, which resulted in a long-lasting decrease in the amplitude of EPSPs. Arrows indicate when a $20 \mathrm{~Hz}$ tetanus (2 s) was applied. Results are comparable to observations from Bliss and Lomo (1973) and Ito and Kano (1982). Baseline spikes at a frequency of $0.2 \mathrm{~Hz}$ were generated by the presynaptic input neuron, in addition to the tetani, to probe the changes in EPSP amplitude. $\boldsymbol{F}$, Simulation of a dual patch clamp STDP experiment. Top, Model setup. A presynaptic neuron is connected to a postsynaptic neuron by an excitatory NMDAR synapse. EPSPs were recorded in the postsynaptic neuron. Two input neurons are connected to the pre- and post-synaptic neurons to control their firing time. STDP simulation results are comparable to observations of STDP in cortical neurons (Froemke et al., 2010). Middle, Potentiation was induced in the model by pre-post firing ( $\Delta t=15 \mathrm{~ms}, 15$ pairings). Bottom, Depression was induced by post-pre pairing ( $\Delta t=-75$ $\mathrm{ms}, 30$ pairings). Baseline spikes were generated at a frequency of $0.125 \mathrm{~Hz}$ by the input neuron connected to the presynaptic neuron to probe the changes in EPSP amplitude.

assumed to be $50 \mathrm{~ms}$. The weights of NMDAR synapses are modified by calcium concentration as follows:

$$
\dot{W}_{j}=\eta\left([\mathrm{Ca}]_{j}\right)\left(\Omega\left([\mathrm{Ca}]_{j}\right)-W_{j}\right)
$$

The $\Omega$ function represents the calcium control hypothesis with $\alpha_{1}$ and $\alpha_{2}$ being $\left[\mathrm{Ca}^{2+}\right]$ thresholds for depression and potentiation induction:

$$
\begin{gathered}
\Omega=\operatorname{sig}\left(\left[\mathrm{Ca}^{2+}\right]-\alpha_{2}, \beta_{2}\right)-\Omega_{\text {rate }} \cdot \operatorname{sig}\left(\left[\mathrm{Ca}^{2+}\right]-\alpha_{1}, \beta_{1}\right), \\
\text { where } \operatorname{sig}(x, \beta)=\frac{e^{\beta x}}{1+e^{\beta x}}
\end{gathered}
$$

The parameter values were originally set as follows: $\alpha_{1}=0.35 \mu \mathrm{M}, \alpha_{2}=0.55 \mu \mathrm{M}, \beta_{1}=\beta_{2}=80 \mu \mathrm{M}$, and $\Omega_{\text {rate }}=0.25$ (Shouval et al., 2002). By setting new $\alpha_{1}$ to 0.1 $\mu \mathrm{M}$ and new $\alpha_{2}$ to $0.4 \mu \mathrm{M}$ (Fig. $2 B$ ), we were able to induce LTD at low input frequency $(1-20 \mathrm{~Hz})$ and post-pre firing $(-15 \sim-5 \mathrm{~ms})$ regardless of the synaptic weights, while LTP was obtained at high input frequency (50-100 $\mathrm{Hz}$ ) and pre-post firing at (5 15 ms), regardless of the synaptic weights (Fig. 2C,D; Table 1). We also changed $\Omega_{\text {rate }}$ from 0.25 to 0.4 , rendering the plasticity more biased toward LTD to stabilize network activity after prolonged sensory exposure.

\section{Individual synapse performance}

After parameter tuning described in the sections above, we simulated the classic tetanus experiment (Bliss and Lomo, 1973) and dual patch clamping experiment
(Markram et al., 1997) on single NMDAR synapses. The synaptic efficacy change successfully reproduced previously published results (Fig. $2 E, F$ ), indicating the reliability of our model at individual synpase level.

\section{Image preprocessing}

For the beard versus no-beard classification simulations, grayscale images of male faces with and without a beard were selected to form two groups, 20 images for each group. Each image was reduced to a $10 \times 10$ pixel pattern (Fig. 1C). For the single face recognition simulations, we used grayscale images of car fronts, dog faces and human faces, 10 images for each category. Each image was converted to a line drawing using edge detection to reduce noise from shading and then downsampled to a $20 \times 20$ pixel pattern (Fig. 1D). For the multi-face recognition simulations, 200 grayscale human face images were selected and each face image was converted to a line drawing and down-sampled to $50 \times 50$ pixels (Fig. 1E). Images were presented to the network by assigning each pixel to a corresponding input neuron and converting pixel values to spike trains. Grayscale values of $0-255$ were mapped to frequencies of $0-50 \mathrm{~Hz}$. Each image stimulus lasted $0.5 \mathrm{~s}$, followed by a silent interval of $0.5 \mathrm{~s}$ (Fig. $1 F$ ) to allow the fading memory to dissipate and prepare the network for the next stimulus.

\section{Fisher's discriminant analysis}

Multiclass Fisher's discriminant analysis was applied to network spiking activity and neuronal spiking activity, to

Table 1. Postsynaptic $\mathrm{Ca}^{2+}$ concentration $[\mu \mathrm{M}]$

\begin{tabular}{lllcccrrr}
\hline Synaptic weight & \multicolumn{1}{c}{ Input frequency [Hz] } & \multicolumn{4}{c}{ Pre-post firing interval [msec] } \\
& 1 & 10 & 20 & 50 & 100 & -15 & -5 & 5 \\
$1 \mathrm{e}^{-8}$ (small) & 0.11 & 0.14 & 0.22 & 0.60 & 1.0 & 0.12 & 0.16 & 0.69 \\
$3 \mathrm{e}^{-8}$ (medium) & 0.15 & 0.19 & 0.30 & 0.85 & 1.0 & 0.17 & 0.21 & 0.78 \\
$6 \mathrm{e}^{-8}$ (large) & 0.27 & 0.32 & 0.52 & 0.90 & 1.0 & 0.29 & 0.33 & 0.93 \\
\hline
\end{tabular}

Points are extracted from Figure $2 \mathrm{C}, \mathrm{D}$, showing $\left[\mathrm{Ca}^{2+}\right]$ under input stimuli of frequency $1,10,20,50$, and $100 \mathrm{~Hz}$, or pre-post firing intervals of $-15,-5,5$, and $15 \mathrm{~ms}$, when initial synaptic weights are small $\left(1 \mathrm{e}^{-8}\right)$, intermediate $\left(3 \mathrm{e}^{-8}\right)$, and large $\left(6 \mathrm{e}^{-8}\right)$. Red indicates LTP and blue indicates LTD. 
obtain Fisher's linear discriminant ratio (FDR). The FDR is calculated as follows:

$$
J(t)=\frac{\sum_{c}\left(\mu_{C}(t)-\mu(t)\right)^{2}}{\sum_{c} \sum_{i \in C}\left(S_{i}(t)-\mu_{C}(t)\right)^{2}}
$$

where $S_{i}(t)$ is the spike count at time bin $t$, in response to stimulus $i ; \mu_{C}(t)$ is the mean spike count at time t for class C stimuli, and $\mu(t)$ is the mean of the class means $\mu_{C}(t)$. The numerator and denominator of the discriminant ratio $\mathrm{J}$ are known as the "between classes scatter" and "within class scatter." A larger value of $J$ indicates better discrimination. More information can be found in Ju et al. (2015).

Network FDR was obtained by summing up the FDRs calculated with network spiking activity in each time bin, serving as an indicator of network separability for input stimuli. Neuronal FDR was calculated with the spiking activity of individual neurons over the entire recording period, serving as an indicator of how informative the neuron is for input discrimination.

\section{Results}

We performed simulations of cortical microcircuits modeled with NMDAR-containing neural networks (see Materials and Methods), to investigate whether they can develop recognition memory. Images were used as sensory inputs and plasticity was enabled during learning and disabled during evaluation of responses to the input images. Simulations typically consisted of three phases: baseline recording, learning and testing. First, to establish a baseline, network responses to all input stimuli were recorded while NMDAR function was disabled. Network firing rate (spikes/second) during the stimulus presentation was calculated and used as a measure of network response. In the second phase, learning was switched on by enabling NMDAR plasticity, and networks were stimulated using only a subset of images, allowing stimulusinduced network spiking activity to alter synaptic strengths. These images should now be "familiar" to the networks. Finally, learning was switched off and network responses to all input stimuli were recorded again. The effect of the learning experience was evaluated for both familiar images presented during the learning phase and the "novel" images, by comparing testing responses with their corresponding baseline values. In all of our simulations, we found that the networks responded differentially to images presented during the learning phase.

\section{Unsupervised classification: beard versus no-beard}

In this first set of simulations, two classes of images were used: human faces of males with and without a beard. In each simulation, only faces from a single class (beard or no-beard) were presented to a network during the learning phase. In each learning round, one face from the selected class was randomly chosen and presented to the network for $1 \mathrm{~s}$. NMDAR plasticity was switched off for testing after each round, and network responses to all faces from both classes were recorded. Figure $3 A$ summarizes the performance of five randomly-generated $10 \times$ $10 \times 5$ networks for 40 rounds of image presentation.
Clearly, networks responded more strongly to faces from the trained class, and the firing rate discrepancy for the two classes increased as more faces were presented. In other words, networks started to display a differential response to the two classes as unsupervised learning took place. This was true whether the beard or no-beard class was used for training. The differential response eventually reaches a steady state, in part because we have set a maximum weight boundary $\left(6.5 \mathrm{e}^{-8}\right)$ for excitatory synapses to prevent overtraining. We also investigated network performance by excluding faces that were presented in the learning phase from the testing. The results showed a similar increase in discrepancy for the responses to the two classes, only with a reduced magnitude (Fig. 3A). In this case, the networks were performing a binary classification task, following unsupervised learning.

\section{Single face recognition}

Next, we performed single face recognition simulations, for which we increased the dimension of the networks to $20 \times 20 \times 5$ and the input layer size to $20 \times 20$. A set of 30 images was used as input stimuli, consisting of 10 car fronts, 10 dog faces, and 10 human faces. The simulations again consisted of three phases: baseline normalization, learning, and testing. NMDAR-dependent plasticity was only switched on during the learning phase. Normalization was performed by recursively adjusting the mean pixel value for each of the 30 images until they evoked comparable baseline responses. In the learning phase, a single human face image was selected from the set of 10 , and presented repetitively for $15 \mathrm{~s}$. NMDAR-dependent plasticity was then switched off for the testing phase, in which we recorded network responses to all images, to determine whether the network responded differently to the selected (learned) face.

For this simulation, we used 30 randomly-generated networks. Instead of limiting the stimuli to human faces, we also conducted trials using images of car fronts and dog faces. For each network, three trials were conducted, each with a randomly selected car, dog, or human image. In total, 90 trials were performed on the 30 networks, and in 84 cases $(93.3 \%)$, the networks exhibited the highest firing rate to the image presented during the learning phase. Examples of network responses before and after the learning phase are shown in Figure $3 B-D$. Testing responses are sorted by firing rate and shown as solid lines, while corresponding baseline responses are plotted as dashed lines. Network firing rate to the images selected for learning significantly increased and became the largest on testing. Responses to novel inputs from the same class as the image selected for learning tended to be elevated from their baseline, although to a lesser extent than the actual learned image. For instance, in the human face learning simulation (Fig. 3D), the network responses to 5 of the 10 human faces (excluding the learned face) were higher than the responses to the dog and car images, suggesting that the network had also generalized to distinguish human faces from dog faces and car fronts. Considering the degree of randomness involved in network construction, and the similarity of the 
A



C

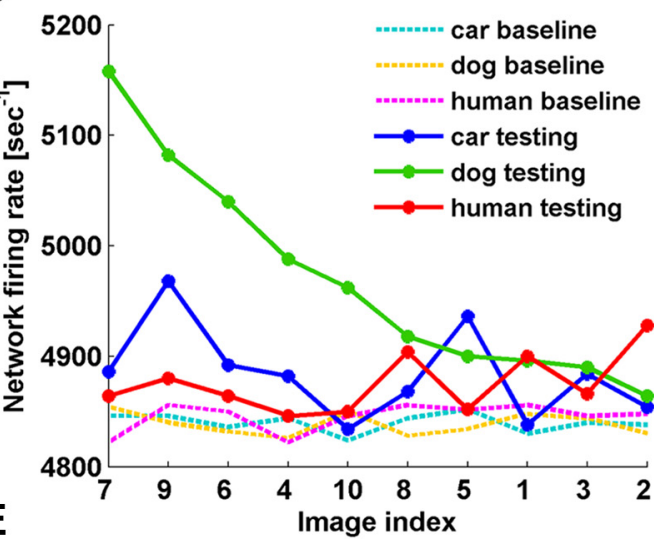

E

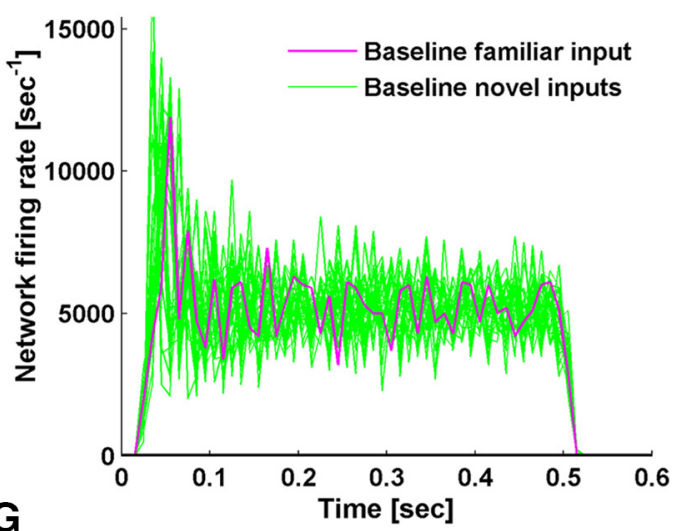

G

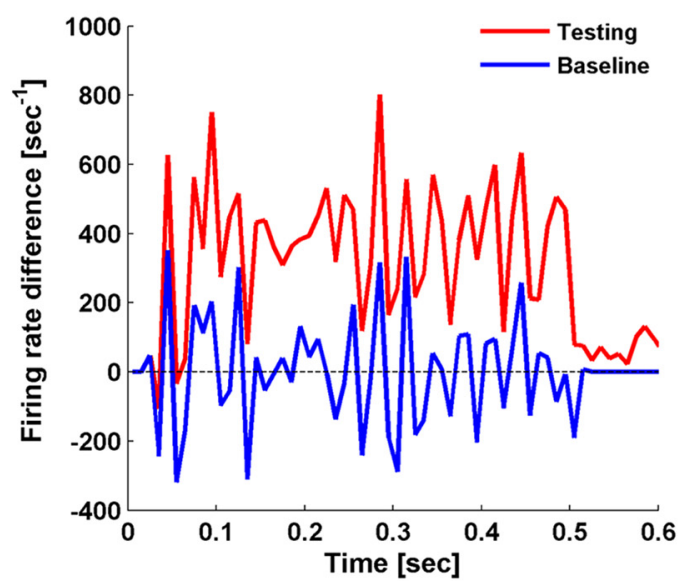

B
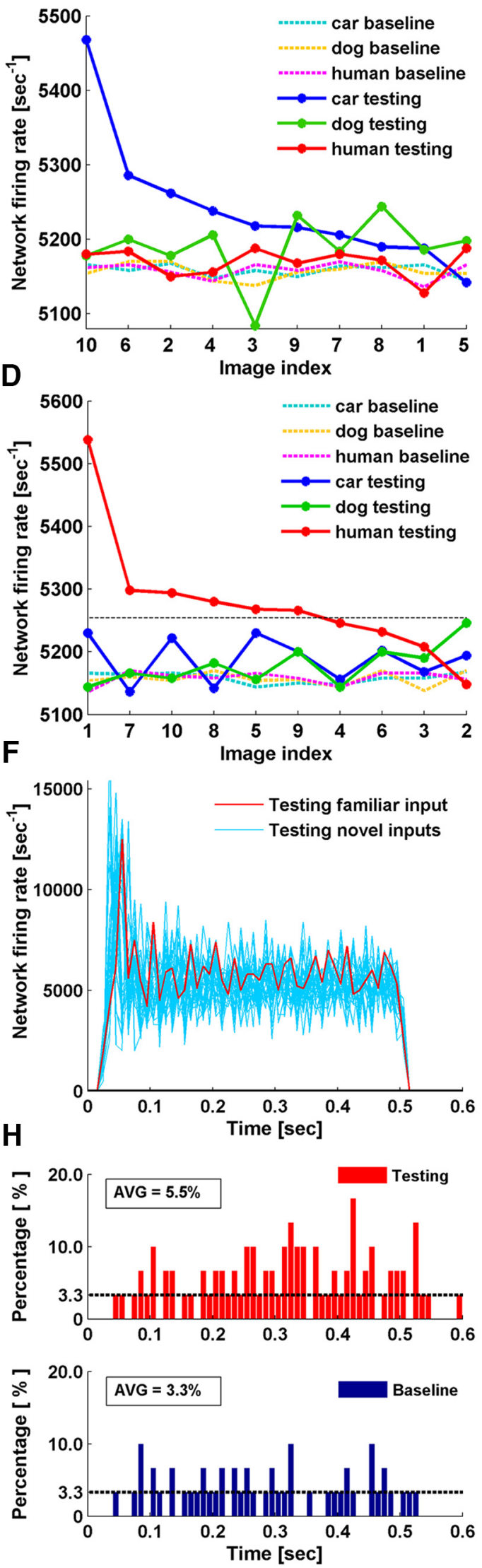

Figure 3. Unsupervised familiarity detection. A, Beard versus no-beard classification. Curves showing the discrepancy in network 


\section{continued}

responses (firing rate $\left[\mathrm{sec}^{-1}\right]$ ) to beard and no-beard faces that develops with sensory exposure to one class. The blue curves represent network response (beard-noBeard) after learning beard faces. The dark blue solid line shows response differences when trained beard faces were used for testing. The light blue dashed line shows the results when untrained beard faces were used for testing, i.e., networks generalizing familiarity to novel beard faces. The red curves represent the results from the same simulation paradigm with no-beard faces used for learning. The red solid line shows response differences when trained no-beard faces were used for testing. The pink dashed line shows the results when untrained no-beard faces were used for testing, i.e., networks generalizing familiarity to novel no-beard faces. Error bars at each time point reflect the SEM of results from five randomly-generated networks. Each network underwent five trials of simulation with randomly-selected face inputs. Curves were normalized by subtracting the discrepancy of network baseline response to the two classes at time 0 . $\boldsymbol{B}-\boldsymbol{D}$, Single face recognition. $\boldsymbol{B}$, The network test responses (solid lines) and corresponding baseline responses (dashed lines) to all 30 images after learning car image 10. Test responses to car images were sorted in descending order. As images of each class were indexed as 1-10, test responses to the other two classes are plotted following the sorted index order. $\boldsymbol{C}$, Network responses to all 30 images after learning dog image 7. $\boldsymbol{D}$, Network responses to all 30 images after learning human face 1. $\boldsymbol{E}, \boldsymbol{F}$, Firing rate dynamics during stimulus presentation of the network shown in $\boldsymbol{D}$. $\boldsymbol{E}$, Network baseline firing rate to all 30 stimuli. $\boldsymbol{F}$, Network firing rate to all 30 stimuli after learning human face 1 . The stimulus of human face 1 is referred as the familiar input. $\mathbf{G}$. Firing rate difference between response to the familiar input and the average of the responses to the novel inputs. The average performance of 10 networks in 30 simulations has been shown. Blue represents the difference in network baseline responses. Red represents the difference in network testing responses, after the single face recognition simulations. $\boldsymbol{H}$, Histograms of time bins where the networks exhibited the highest firing rate to the familiar input. Network firing rate was obtained as in $\boldsymbol{E}, \boldsymbol{F}$, binning was at $10 \mathrm{~ms}$. Time bins where networks exhibited the highest firing rate to the familiar input were identified. The number of the identified time bins was summed for 30 simulations carried out 10 networks, and the percentage was calculated with respect to 30 simulations, with chance level being 1/30 (3.3\%).

images within each class, we conclude that networks of $20 \times 20 \times 5$ neurons can learn to detect a familiar image with high specificity.

The network responses in Figure $3 B-D$ are network firing rates averaged over the entire recording period. In fact, the dynamic network firing rates plotted as a function of time underwent initial rising phases and declined to steady state phases (Fig. 3E,F). By solely looking at firing rate difference, network response to the familiar input seems to have increased over the entire recording period after learning (Fig. 3G). Whether the increase in individual time bins is sufficient for familiarity detection is further analyzed. If we assume the networks are able to detect familiarity in the time bins where networks exhibited the largest firing rate to the familiar input, we can count the number of such time bins and compare it before and after learning. The results show that there is no specific time window that clearly separates the familiar input from the novel inputs, but the separation gets better in the later stage of stimulus presentation (Fig. $3 H$ ).

\section{Multi-face recognition}

Now that these relatively small neural networks have demonstrated they can discriminate a single familiar face from many novel ones, we tested whether networks can perform familiarity detection to more than one face. We began with presenting two human face stimuli to a single network, using the same set of 30 images and $20 \times 20 \times$ 5 networks. Unlike the high accuracy (93.3\%) of the single face recognition simulations, familiarity detection accuracy for two face stimuli dropped to $70.0 \%$ (data not shown). This performance decline informs us of the limited capacity of $20 \times 20 \times 5$ networks.

We therefore increased dimensions of the networks to $50 \times 50 \times 5$ and input layers size to $50 \times 50$. In addition, the resolution of the 30 images was increased to $50 \times 50$ pixels, so that their dimension matched that of the input layer. With these larger networks, the accuracy of famil- iarity detection for two face stimuli increased to $96.7 \%$. The performance improvement could be due to either the increase in network size or the increase in resolution of the face images. To distinguish between these two options, we repeated the simulations on $50 \times 50 \times 5$ networks, but with the original low-resolution $20 \times 20$ pixel image stimuli as inputs. Each pixel in the $20 \times 20$ images was replicated once to create images of $40 \times 40$. One column and one row of void pixels were then added to the right and bottom of the matrices to make images of dimension $50 \times$ 50 . When these low-resolution $50 \times 50$ stimuli were presented to the networks, the accuracy of familiarity detection remained at $96.7 \%$ (Fig. 4A), suggesting that increased network dimension was responsible for the accuracy improvement.

Interclass learning of three image classes (car + dog + human) using $50 \times 50 \times 5$ networks also produced stable and accurate results (data not shown). For multiple images, we presented each image for $15 \mathrm{~s}$ and then moved to the next image. In contrast, we also tried looping through all images 15 times. The first presentation protocol resulted in slightly better accuracy, and was used for all the remaining simulations.

To further evaluate the limits of these neural networks on multi-face recognition, we converted a database of 200 human face images to $50 \times 50$ pixel stimuli. In each simulation, a randomly-generated $50 \times 50 \times 5$ network was presented with 10 human face images, each for $6 \mathrm{~s}$, and testing was conducted with the 10 learned faces plus 10 novel faces drawn randomly from the database. We used $6 \mathrm{~s}$ instead of 15 , to prevent overtraining of the network. After sorting the network firing rate for all 20 faces, a hypothetical threshold was drawn between the responses to faces ranking 10th and 11th, to evaluate the ability of the networks to separate familiar and novel faces. This procedure is similar to the empirical ranking theory of vision (Purves et al., 2011), which suggests that subjects perceive the relative color and brightness of 
A
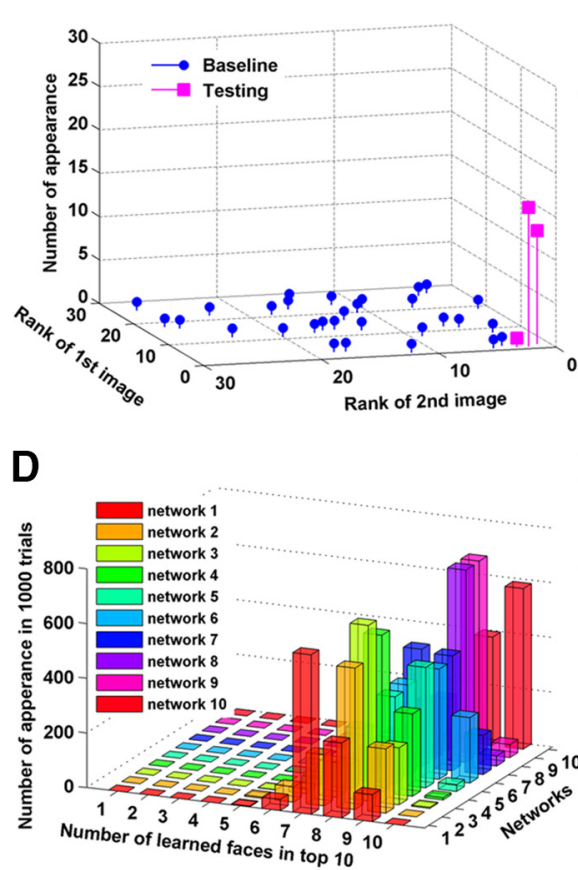

G
B

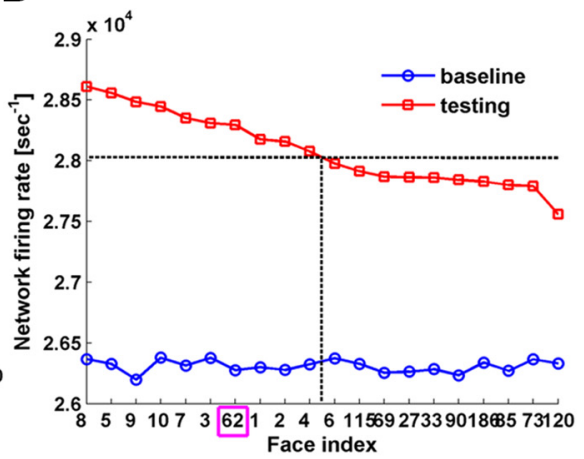

E

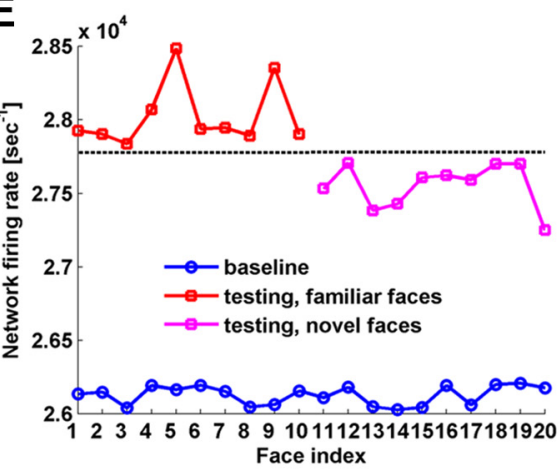

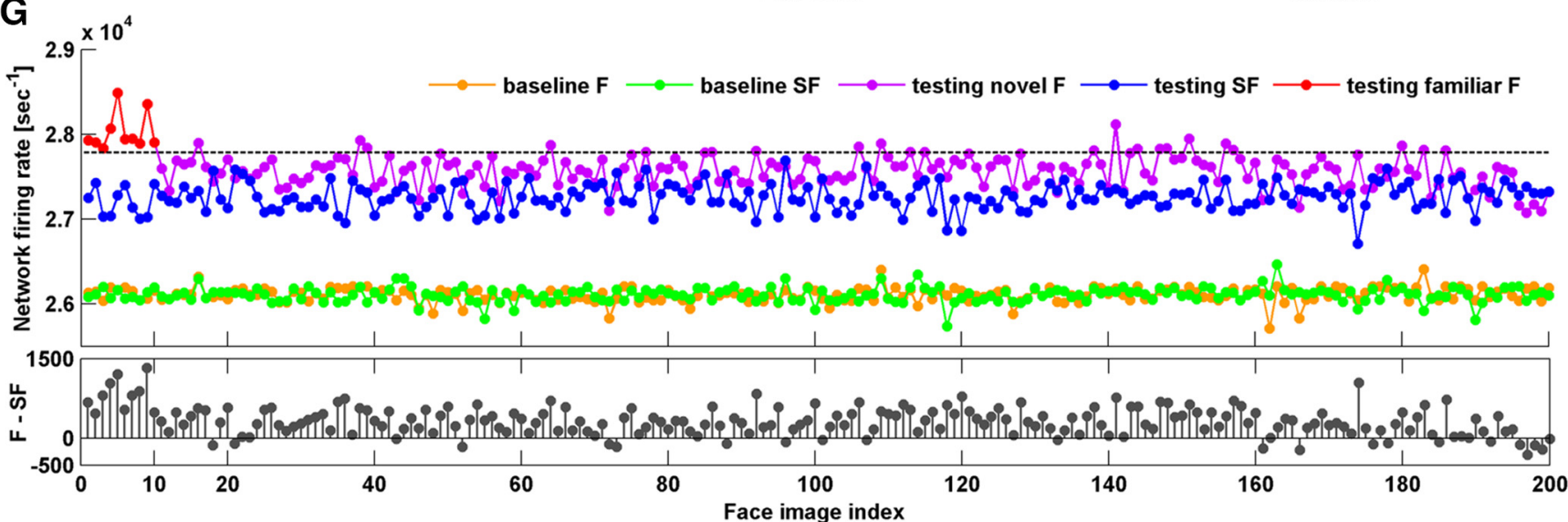

C

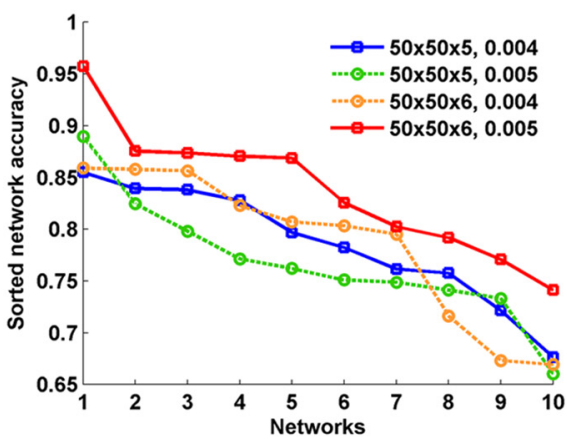

F

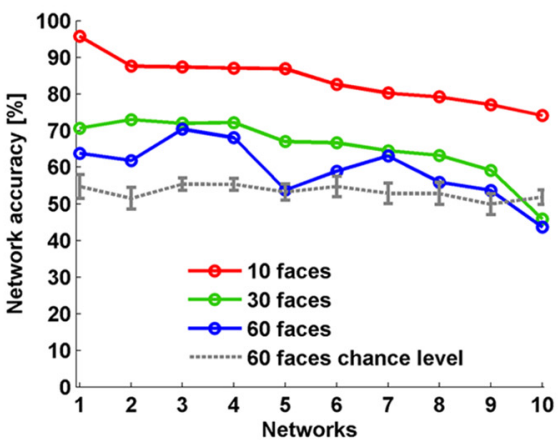

Figure 4. Familiarity detection for multiple faces. $\boldsymbol{A}$, Results of $50 \times 50 \times 5$ networks learning two human faces. Each bar is located at the point whose coordinates represent the rank of network firing rate for the two learned faces. The height of each bar reflects the number of occurrences of such a combination (30 in total). Blue and magenta represent network baseline and testing response, respectively. Note that after learning, the two familiar faces ranked 1 and 2 in 29 of 30 trials and 1 and 3 in the remaining trial. $\boldsymbol{B}-\boldsymbol{E}$, Results of learning 10 faces and testing them against 10 novel faces randomly drawn from a database of 200 faces. $\boldsymbol{B}$, Results of a $50 \times 50 \times 5$ network learning 10 faces. The curves show baseline and sorted testing response after learning faces 1-10. The network ranks nine familiar faces among the top 10, with only one outlier (face 62, highlighted in magenta). The accuracy of this network therefore was $9 / 10$ or $90 \%$. C , The effect of network dimension and Cscale value was evaluated by performing 1000 trials for 10 different networks (see Results). For each trial, the accuracy was calculated as in $\boldsymbol{B}$. The curves illustrate the accuracy (averaged over 1000 trials) for the 10 networks with dimensions and Cscale values indicated in the legend. $\boldsymbol{D}$, Accuracy distribution of 1000 testing trials conducted on the ten $50 \times 50 \times 6$ networks with Cscale 0.005 . $\boldsymbol{E}$, Baseline and testing response of the best-performing $50 \times$ $50 \times 6$ network after learning face 1-10. Network firing rate differentiates familiar faces completely from novel faces. $\boldsymbol{F}$, Network average accuracy of the $50 \times 50 \times 6$ networks after learning 10 faces (red) and the corresponding accuracy after learning 30 faces (green), 60 faces (blue), and chance level at 60 faces (gray, dotted line). Error bars are the SDs of 1000 trials. G, top, Baseline and testing response of the best-performing $50 \times 50 \times 6$ network to all 200 faces and their scrambled versions, after learning face $1-10$. F, faces; SF, scrambled faces. Bottom, Pairwise network firing rate difference to faces and scrambled faces (F-SF).

objects by internally ranking the empirical brain activity they evoke. In our case, the percentage of learned faces appearing in the top 10 reflects familiarity detection accuracy. The 10 faces used for presentation to the net- works were randomly selected from the database, indexed as $1-10$ and fixed for all simulations. The 10 novel faces were randomly drawn from the remaining database and were varied for each testing trial. Figure $4 B$ shows the 
Table 2. Statistical table

\begin{tabular}{|c|c|c|c|}
\hline Network & Data structure & Type of test & $p$ value \\
\hline 1 & Normality test: failed $(p<0.05)$ & Mann-Whitney rank sum test (one-side) & $5.29 e^{-315}$ \\
\hline 2 & Normality test: failed $(p<0.05)$ & Mann-Whitney rank sum test (one-side) & $3.45 e^{-322}$ \\
\hline 3 & Normality test: failed ( $p<0.05)$ & Mann-Whitney rank sum test (one-side) & 0.00 \\
\hline 4 & Normality test: failed $(p<0.05)$ & Mann-Whitney rank sum test (one-side) & 0.00 \\
\hline 5 & Normality test: failed $(p<0.05)$ & Mann-Whitney rank sum test (one-side) & 0.03 \\
\hline 6 & Normality test: failed $(p<0.05)$ & Mann-Whitney rank sum test (one-side) & $5.16 e^{-158}$ \\
\hline 7 & Normality test: failed $(p<0.05)$ & Mann-Whitney rank sum test (one-side) & $2.42 e^{-322}$ \\
\hline 8 & Normality test: failed $(p<0.05)$ & Mann-Whitney rank sum test (one-side) & $3.71 e^{-91}$ \\
\hline 9 & Normality test: failed $(p<0.05)$ & Mann-Whitney rank sum test (one-side) & $1.49 e^{-140}$ \\
\hline 10 & Normality test: failed $(p<0.05)$ & Mann-Whitney rank sum test (one-side) & 1.00 \\
\hline
\end{tabular}

sorted responses and hypothetical threshold for a network that was able to detect 9 out of the 10 presented faces in one testing trial, and therefore was considered $90 \%$ accurate for this trial. The average accuracy of a network was measured by averaging the results of 1000 testing trials. Performance of ten randomly-generated 50 $\times 50 \times 5$ networks for the above simulations is shown in Figure $4 C$, blue. The best network reached $\sim 85 \%$ accuracy on average for 10-face familiarity detection.

In an attempt to further improve the performance, we generated a different set of ten randomly-generated networks, with one more layer added to the network reservoir $(50 \times 50 \times 6)$, and increased the connection probability from the input layer to the reservoir (Cscale $=0.004$ to Cscale $=0.005)$. The resulting network average accuracy is shown in Figure $4 C$, red. Not only did the overall accuracy improve, but also a network with $>95 \%$ accuracy emerged, indicating the network was able to detect all 10 familiar faces accurately for at least half of the 1000 testing trials. Figure $4 D$ summarizes the accuracy distribution of 1000 testing trials for the ten $50 \times 50 \times 6$ networks. The distribution is significantly shifted from what would be expected by chance alone, i.e., five familiar faces falling in the top 10 . Figure $4 E$ plots the response of the best-performing $50 \times 50 \times 6$ network for one of the testing trials. Unsupervised learning has clearly modified the network response and a hypothetical threshold can be drawn which accurately differentiates familiar from novel faces.

We also evaluated the performance of ten randomlygenerated $50 \times 50 \times 5$ networks with Cscale 0.005 and ten randomly-generated $50 \times 50 \times 6$ networks with Cscale 0.004 (Fig. 4C, dotted lines). Neither achieved comparable accuracy as $50 \times 50 \times 6$ networks with Cscale 0.005. From these results, it seems that both the network size and the number of input connections are important. An increase of input connections together with a larger network results in larger available network space for better familiarity storage.

In fact, the network capacity of multi-face recognition is not restricted to 10 faces. In Figure $4 F$, we plot the accuracy of the ten $50 \times 50 \times 6$ networks after learning 10 faces (red), 30 faces (green), and 60 faces (blue), as well as the chance level for 60 faces (gray). To our surprise, only two networks performed equivalently to or below their corresponding chance levels after learning 60 faces. The remaining eight networks performed signifi- cantly better than chance levels (Table 2, $n=1000, p<$ 0.01). Accuracy after learning 30 and 60 faces was measured by the percentage of familiar stimuli appearing above the hypothetical threshold (set as below $50 \%$ of the population) after pooling the same number of novel stimuli with familiar stimuli. The chance level at 60 faces was calculated by the percentage of familiar stimuli appearing above the hypothetical threshold after sorting network baseline firing rates to the familiar and novel stimuli.

In addition, we generated scrambled versions of the $50 \times$ 50 face images from the same database by relocating all pixels to new random $(x, y)$ positions, and recorded the network responses to them after learning 10 human faces. The results show that networks not only discriminated the familiar faces from the novel faces, but also could discern novel faces from their scrambled versions (Fig. 4G). The trained networks appeared to have acquired the concept of a "face" and responded less to scrambled faces.

\section{Synaptic weight change}

Given that synaptic weight changes underlie the observed results described above, we plot in Figure 5 the network weight changes in three scenarios: weight change after exposure to a human face stimulus for 1, 3, and $5 \mathrm{~s}$ (Fig. 5A); weight change after exposure to a car, a dog or a human face image each for $15 \mathrm{~s}$ (Fig. 5B); and weight change after exposure to multiple face images (Fig. 5C). A common finding emerges for all three scenarios: unsupervised learning caused a subset of synapses to be potentiated, and a different but larger subset of synapses to be depressed. The amplitude of potentiation is larger than the amplitude of depression, on average. This is in line with a proposed familiarization mechanism that a small subset of neurons becomes strongly responsive after sensory exposure while depression occurs pervasively among other neurons, sharpening the familiarity response (Freedman et al., 2006; Meyer et al., 2014). Figure $5 D$ shows the time course of the change in synaptic strength for nine representative synapses randomly picked from a $50 \times 50 \times 6$ network during the exposure to 10 faces. Synaptic weights changed in unique ways in response to each face stimulus.

\section{Subnetwork formation}

If we put together the phenomena of how synaptic weights change after sensory exposure and how available network space affects accuracy, a theory emerges that 
A
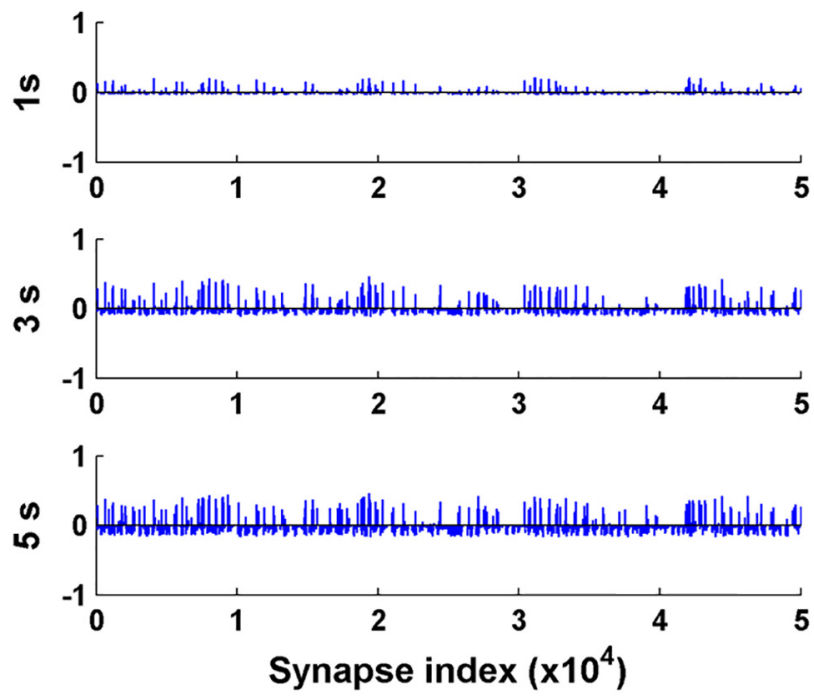

C


B
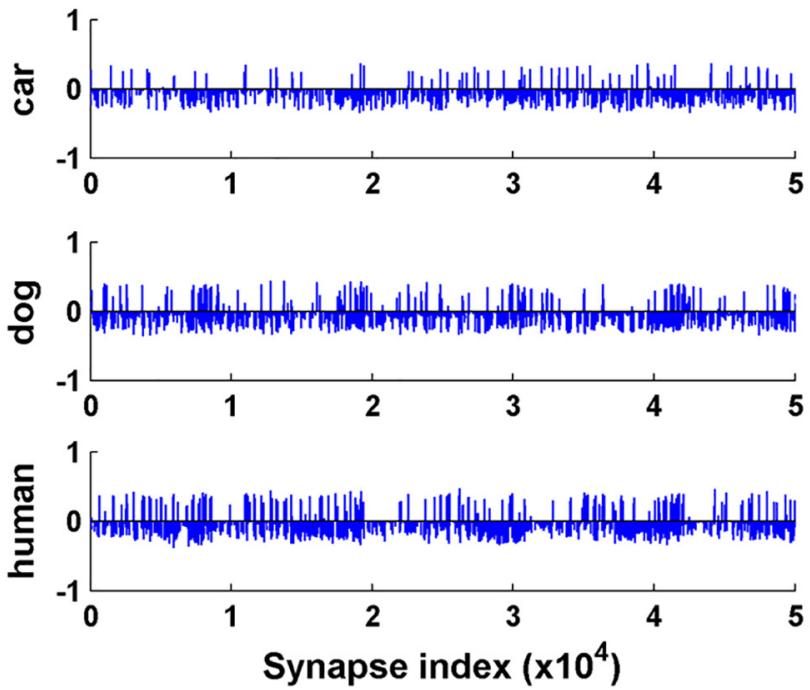

D

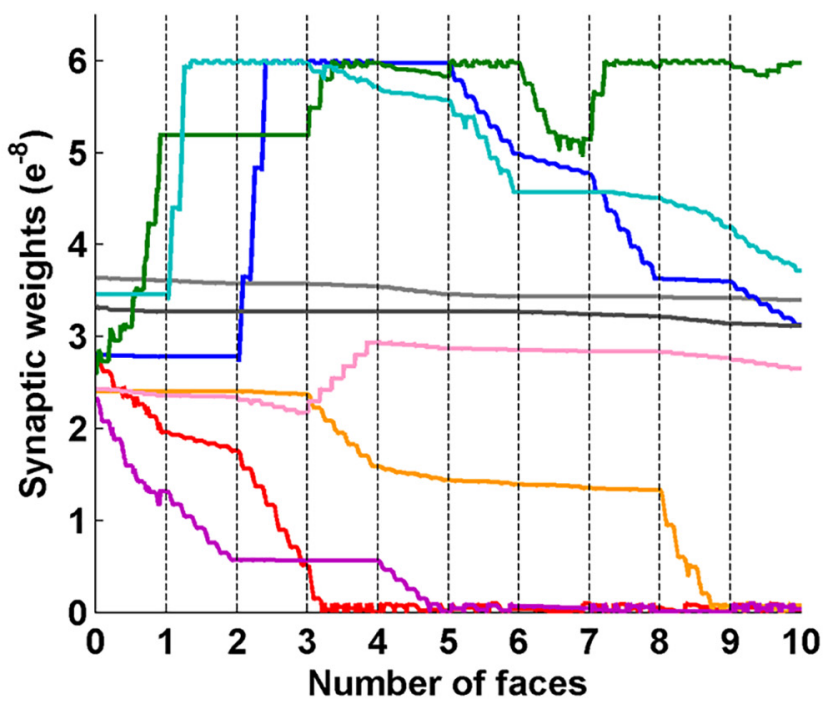

Figure 5. Synaptic weight changes caused by learning. $\boldsymbol{A}$, Network synaptic weight change after the presentation of 1,3 , and $5 \mathrm{~s}$ of the same human face stimulus, in comparison to initial synaptic weights. $\boldsymbol{B}$, Network synaptic weight change after learning a car, dog, or human stimulus, each for $15 \mathrm{~s}$, in comparison to initial synaptic weights. $\boldsymbol{C}$, Network synaptic weight change after learning 1 , 5 , and 10 human face image stimuli, each for $6 \mathrm{~s}$, in comparison to initial synaptic weights. $\boldsymbol{A}-\boldsymbol{C}$, Only a subset of NMDAR synapses in the network are shown for clarity and the $y$-axis scales are $\pm 1 \mathrm{e}^{-7}$. $\boldsymbol{D}$, Time course of weight change of nine randomly-selected NMDAR synapses during the exposure to ten faces, each for $6 \mathrm{~s}$.

could possibly address the stability-plasticity dilemma (Grossberg, 1980, 2013; Mermillod et al., 2013). Whenever a new image stimulus enters the network reservoir, an image-representative subnetwork is formed by potentiated and depressed synapses. Providing the network reservoir is large enough and has sufficient complexity, a large number of image-representative subnetworks that differ slightly from each other can co-exist in the network. This reduces the chance that formation of a new memory overwrites an older one, allowing each subnetwork to support familiarity detection. To provide support for this idea, we identified the set of synapses whose weights were modified after the stimulus presentation. In Figure $6 A, B$, the potentiated synapses are highlighted in red and green, respectively, for the same network after exposure to two different face images. In Figure $6 C$, the potentiated synapses are highlighted after the same network was exposed to both face images. Notice the difference between the two image-representative subnetworks, and how they can co-exist in the network reservoir. These subnetworks may act as neural clusters carrying memory traces (Liu et al., 2012). In Figure 6A-E, the subnetworks are viewed from the top. As the faces resemble each other to a certain degree, the corresponding subnetworks are proximate to each other. They are also densely connected through the layers, as seen in a side view (Fig. 6F).

Following this idea, we plotted the potentiated synapses of the ten $50 \times 50 \times 6$ networks after learning 

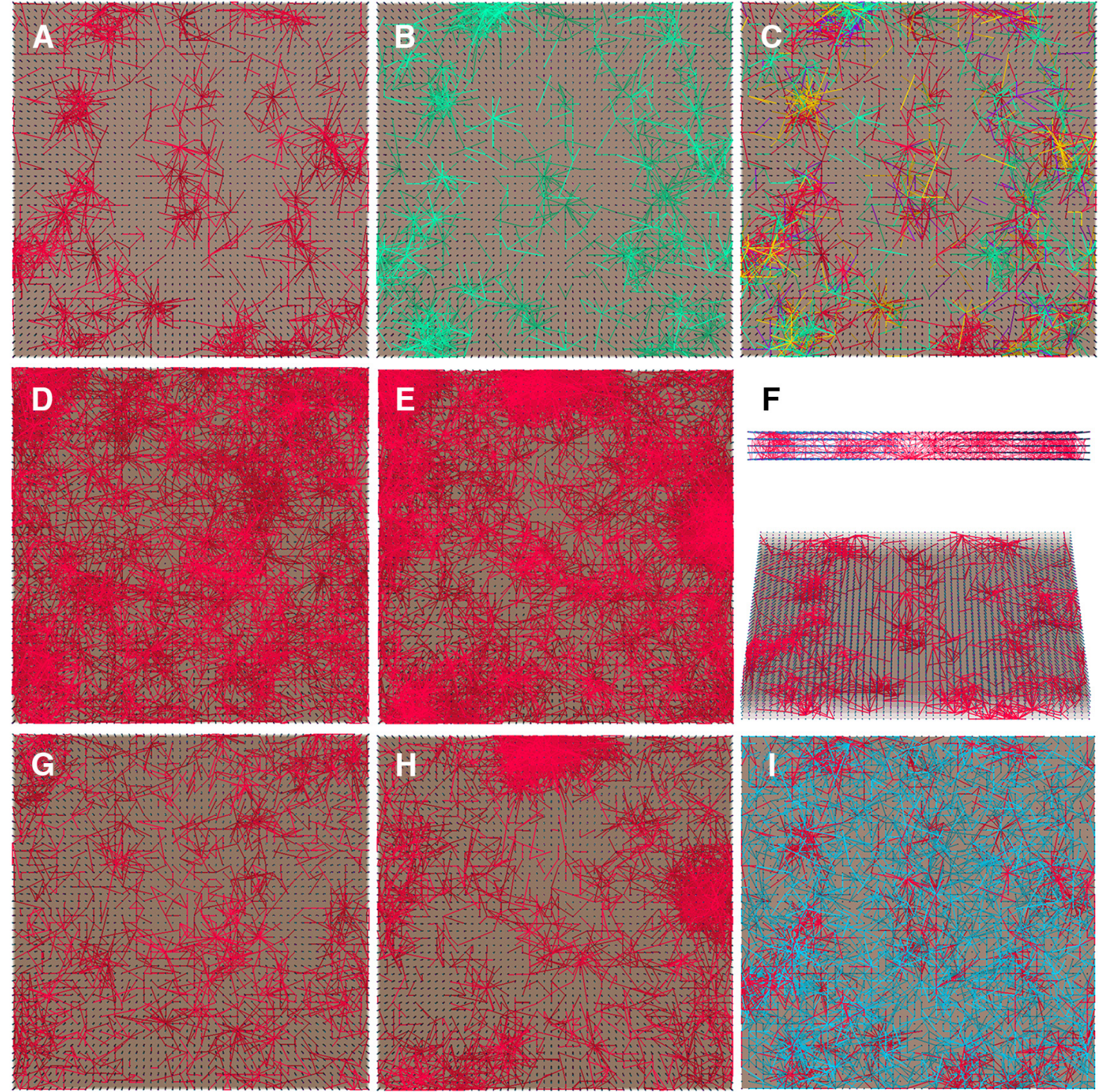

Figure 6. Importance of subnetwork topology. $\boldsymbol{A}-\boldsymbol{C}$, Colored lines indicate potentiated synaptic connections of a $50 \times 50 \times 6$ network after learning face $1(\boldsymbol{A})$, face $2(\boldsymbol{B})$, or face 1 and $2(\boldsymbol{C})$. Yellow highlights the synapses in $\boldsymbol{C}$ that also appeared in both $\boldsymbol{A}$ and $\boldsymbol{B}$; red and green highlights the synapses that only appeared in $\boldsymbol{A}$ and $\boldsymbol{B}$, respectively. Purple indicates the synapses that newly appeared after learning both faces. $\boldsymbol{D}, \boldsymbol{E}$, Potentiated synaptic connections in the networks with the best $(\boldsymbol{D})$ and worst $(\boldsymbol{E})$ accuracy after learning 60 faces. $\boldsymbol{F}$, Side views of the potentiated synaptic connections in $\boldsymbol{A}$. $\boldsymbol{G}$, Potentiated synaptic connections of the network in $\boldsymbol{D}$ after learning 60 faces, in comparison to synaptic weights obtained after learning 30 faces. $\boldsymbol{H}$, Potentiated synaptic connections of the network in $\boldsymbol{E}$ after learning 60 faces, in comparison to synaptic weights obtained after learning 30 faces. $\boldsymbol{I}$, Potentiated (red) and depressed (blue) synapses after learning face 1, for the same simulation shown in $\boldsymbol{A}$. Thresholds for significant weight increase and decrease are $1 \mathrm{e}^{-8}$ and $-1 \mathrm{e}^{-8}$ for all panels.

multiple faces. Figure $6 D, E$ shows the potentiated synapses in the two networks with the best and the worst accuracy, after exposure to 60 faces (Fig. $4 F$, network 3 and 10 , respectively). Dense clusters can be seen in the poorly-performing network (Fig. 6E). We also investigated the evolution of subnetworks from 30- to 60-face exposures. Figure $6 G, H$ graphs the incremented subnetworks corresponding to $D$ and $E$. Newly potentiated synapses are spread throughout the reservoir in the network with high accuracy, whereas newly potentiated synapses are clustered in the network with low accuracy. We calculated the average clustering coefficients (Watts and Strogatz,
1998) of the formed subnetworks for the 10 networks after learning 10,30, and 60 faces, and correlated the average clustering coefficients with the corresponding network accuracies. The data suggest that strong clustering of subnetworks negatively affects network performance ( $r=$ $-0.80, p=9.05 \mathrm{e}^{-8}$ ). In general, subnetworks were more distributed in networks with better accuracy, and more aggregated in networks with poorer accuracy. One possible explanation is that overlapping subnetworks lead to increased chance for existing memories to be overwritten. The dense clustering observed in poorly performing networks might be caused by local densely-connected neu- 
rons, which tend to self-potentiate excessively due to recurrent pathways, thereby reducing memory capacity.

For the network with the best accuracy, we attempted to permute the initial weights of all NMDAR synapses of the network and repeated the same simulation of the 60-face exposure. After permutation, the network accuracy failed to remain the best. It seems that the preimposed network circuitry, which is determined by the initial synaptic connection weights, is another important factor for network performance.

\section{Separability}

In addition to using network firing rate as a readout for familiarity detection, we applied Fisher's discriminant analysis to search for other features that may help define familiarity. Ten $20 \times 20 \times 5$ networks and the single face recognition simulations were used for the analysis. Network response to the familiar stimulus is referred to as the familiar response, and responses to the remaining 29 stimuli are referred to as control responses. FDR is calculated between two classes (see Materials and Methods). Specifically, familiar FDR is calculated by labeling the familiar response as Class I and the control responses as Class II. Control FDRs are calculated by labeling one of the control responses as Class I and the remaining control responses together with the familiar response as Class II. Baseline FDRs are calculated in the same way but with network baseline responses to all 30 image stimuli. Baseline FDR values represent how well each image stimulus is separated from others, before learning.

Compared with the corresponding baseline FDRs, we see a general increase for both familiar FDR and control FDRs after sensory exposure (Fig. 7A). In 26 out of 30 cases, familiar FDRs show the largest magnitude of relative increase from baseline compared with control FDRs; and in 22 out of 30 cases, familiar FDRs have the largest final values. Larger familiar FDRs indicates that the network discriminated the familiar better from the control stimuli after learning, implying greater separability at the network level. Figure $7 B$ shows the network FDRs calculated in each time bin. The larger familiar FDR can be explained by the larger FDR values in each time bin. Sensory exposure has also extended the network separability to beyond the stimulus presentation window (0-0.5 s).

To further understand the changes occurring at the neuronal level that supported familiarity detection, we applied the analysis to individual neurons. Similarly, familiar FDR is calculated by labeling the neuronal response to the familiar stimulus as Class I and responses to control stimuli as Class II; control FDRs are calculated by labeling the neuronal response to one of the control stimuli as Class I and responses to the remaining control stimuli and the familiar stimulus as Class II.

Compared with corresponding baseline FDRs, neurons that show increased FDRs to the familiar stimulus are considered as critical and potentially correlate with the emergence of familiarity. We noticed that the neurons with significantly increased FDRs tended to evolve more often from the neurons with negligible baseline FDRs (Fig. 7C). Neuronal baseline FDR was found to be negatively corre- lated with FDR increase $\left(r=-0.30, p=4.46 \mathrm{e}^{-164}\right)$. The top-ranking neurons showed little response at baseline but increased firing rate after sensory exposure. The low baseline response implies that they did not receive much information from the input layer initially, or equivalently, that they were not wired to the input-responding pathway before the training. The increase in firing rate after learning implies that their connections to the input-responding pathway were strengthened. These critical neurons are recruited by unsupervised learning to the subnetwork that responds to a specific stimulus. Once recruited, they may also fire to other nonlearned inputs, but their response to the familiar input is stronger (Fig. 7D).

So far, we have looked at the formation of subnetworks (altered connections) and the emergence of critical neurons for familiarity detection. The intersection between the two intrigues us. Therefore, we investigated whether the critical neurons belong to the subnetworks. The subnetwork in Figure $6 A$ was analyzed. We selected 200 critical neurons with top-ranking familiar FDR values and 118 of them belong to the subnetwork (Fig. 7E). If 200 neurons were randomly selected from the network reservoir, we would expect $\sim 28$ neurons to overlap with the subnetwork by chance. For comparison, we also selected 200 critical neurons with top-ranking control FDR values for control faces 2-10 for the same network, after learning face 1. The intersections with the subnetwork dropped to $43 \pm 8$ (mean \pm SD) neurons (Fig. 7F). The observation held when other faces were used for learning. Therefore, the critical neurons selected for the learned face and the subnetwork formed after exposure are highly correlated. Their colocalization in space further supports the idea that the potentiated subnetwork is an important memory storage unit.

\section{Effect of STDP and LTD/LTP}

As the NMDAR-based plasticity we implemented supports both LTD/LTP and STDP mechanisms, we investigated how they contributed to familiarity detection. Learning in which LTP/LTD and STDP worked together stabilized network performance efficiently to the optimum accuracy (Fig. 8A, STDP \& LTP/LTD). Then we selectively deactivated STDP by removing BPAPs (Eq. 4) and repeated the single face recognition simulations on 10 networks. The results showed a severe reduction of accuracy (Fig. 8A, LTP/LTD). At later time points during learning without STDP, network accuracy reversed and networks responded less to the familiar input than to novel inputs on average. This is the result of an LTD-dominant $\Omega$ function (Eq. 10) tuned to account for the effect of BPAPs. To know what LTP/LTD alone is capable of, we need to readjust the parameters of the $\Omega$ function to compensate for the loss of BPAPs (Fig. 8A, LTP/LTD adjusted, $\alpha_{1}=$ $0.3, \alpha_{2}=0.4$ and $\left.\Omega_{\text {rate }}=0.3\right)$. Comparing the curves of STDP \& LTP/LTD, and LTP/LTD adjusted (Fig. 8A), we postulate that the effect of STDP is to increase learning specificity.

Does this mean that STDP alone could be sufficient for familiarity detection? As STDP cannot be isolated to function alone under the calcium control hypothesis, we 
A


C $2 \times 10^{11}$
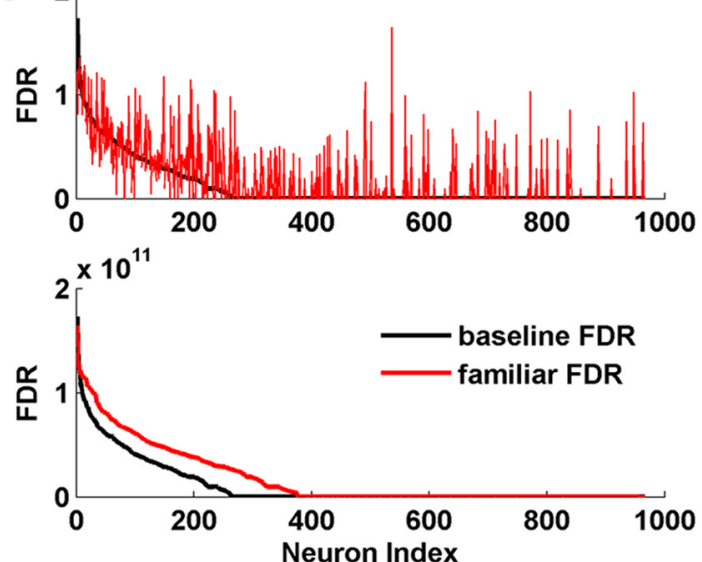

E

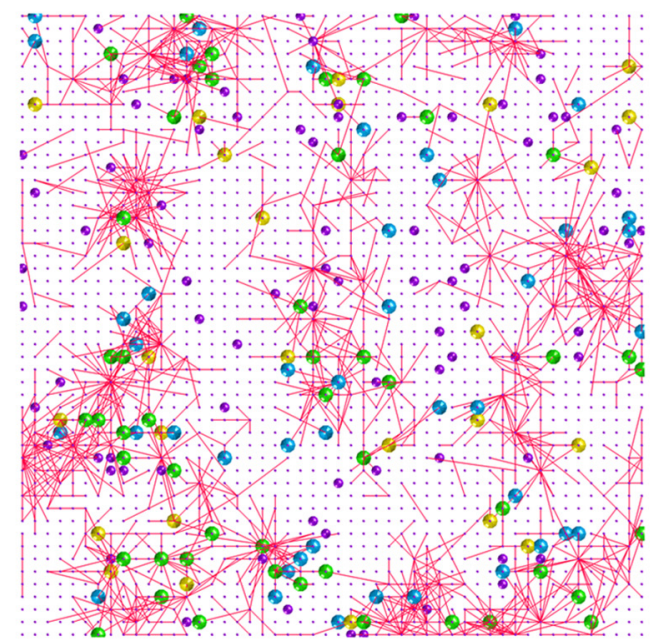

B $6 \times 10^{11}$
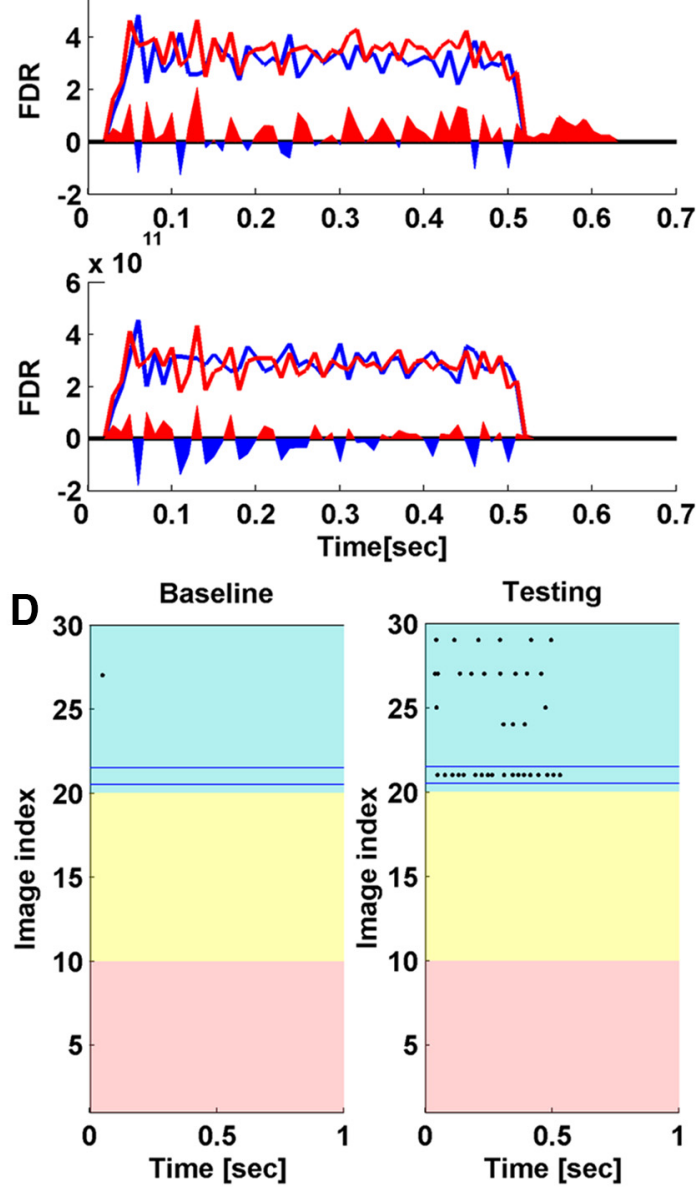

$\mathbf{F}$

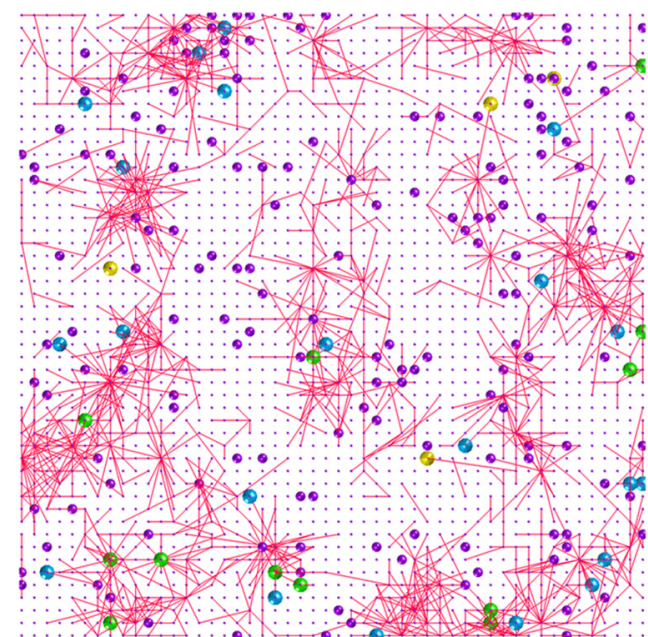

Figure 7. FDR analysis. $\boldsymbol{A}-\boldsymbol{D}$, FDRs were calculated for the $20 \times 20 \times 5$ network after the single face recognition simulation shown in Figure 3D. The input stimulus was human face 1, indexed as image 21 in the set of 30 test images. A, top, Network FDRs after learning human face 1. Bottom, Network FDR relative changes from baseline after learning human face 1. Red dashed lines represent the maximum in the group. Green dashed lines represent the presented stimulus. Network FDR has the largest value to the familiar stimulus after learning, and the largest relative increase from baseline. B, Network FDRs calculated with network activity in 10-ms time bins. Red lines show the FDRs to the familiar stimulus (image 21) and blue lines show the FDRs to one of the control stimuli (image 9). The filled area shows the difference between the two, with red indicating a larger value of the red line and blue indicating a larger value of the blue line. Top, The FDRs calculated based on network response after sensory exposure. Bottom, the FDRs calculated based on network baseline response. $\boldsymbol{C}$, Neuronal familiar FDRs were calculated for the active neurons (recorded spikes $>0$ ) after the same simulation. Top, Black indicates the sorted baseline neuronal FDRs, and red indicates the familiar FDRs of the correspond- 


\section{continued}

ing neurons after the simulation. Bottom, black indicates the sorted baseline neuronal FDRs, and red indicates the sorted neuronal familiar FDRs. $\boldsymbol{D}$, Baseline and testing responses to all stimuli of the neuron with the largest FDR increase in $\boldsymbol{C}$. Red, yellow, and blue indicate responses to car, dog, and human stimuli. Neuronal baseline response is negligible, indicating an inactive role in the network. After sensory exposure, neuronal firing rate to image 21 was greatly increased. The response also generalized to other human face images (index 21-30). $\boldsymbol{E}, \boldsymbol{F}$, FDRs were also calculated for neurons in the $50 \times 50 \times 6$ network after the simulation shown in Figure $6 A$. The input stimulus was human face 1 from the 200 -face database. The subnetwork in Figure $6 A$ connects 1728 unique neurons, including 187 presynaptic neurons, 1238 postsynaptic neurons, and 303 neurons that are both pre- and postsynaptic. $\boldsymbol{E}$, Intersection of top 200 FDR neurons with the formed subnetwork $(n=118)$. Yellow, blue, and green indicate those FDR neurons that overlapped with presynaptic neurons ( $n=26)$, postsynaptic neurons $(n=43)$, and both pre- and postsynaptic neurons $(n=49)$ in the subnetwork. Purple indicates nonoverlapped FDR neurons $(n=82)$. $\boldsymbol{F}$, Intersection of top 200 FDR neurons calculated for control face 2 with the formed subnetwork after the network was exposed to face $1(n=37)$. Yellow, blue, and green indicate those FDR neurons that overlapped with presynaptic neurons $(n=4)$, postsynaptic neurons $(n=21)$, and both pre- and postsynaptic neurons $(n=12)$ in the subnetwork. Purple indicates nonoverlapped FDR neurons $(n=163)$.

repeated the single face recognition simulations using the STDP synapse model available in the CSIM package, which is based on the work by Froemke and Dan (2002) and Gutig et al. (2003). We adopted the same parameter values used in Song et al. (2000) and Xue et al. (2013). For comparison, we used the same 10 networks, replacing the NMDAR synapses with STDP synapses. Initial synaptic weights were kept the same to ensure that the networks' baseline responses to the input stimuli were comparable to the previous values obtained with NMDAR synapses. Then we switched on the STDP plasticity and repeated the single face recognition simulations (Fig. $8 \mathrm{~A}$, STDP') . Learning accuracy of networks with STDP-only synapses improved gradually, but it did not reach the optimum value (rank 1) on average after $15 \mathrm{~s}$ of learning. The NMDAR model which incorporates both STDP and LTP/LTD was more accurate and more efficient. The network response relative difference after learning with STDP-only synapses was also much smaller than with NMDAR synapses (Fig. 8B).
Note that the STDP mechanism, which is part of the NMDAR synapses, works through calcium concentration, whereas the STDP synapse in CSIM works through an equation which uses the time intervals between presynaptic spikes and postsynaptic spikes as inputs. On average, STDP-only plasticity requires repetitive presentation of the stimulus to develop accurate familiarity. While with NMDARdependent plasticity, networks develop familiarity fast and efficiently, as required for one-shot learning (Yakovlev et al., 2008). Therefore, it seems that the calcium control hypothesis is an efficient way of combining the frequency rule (LTP/LTD) and the timing rule (STDP), enhancing both forms of plasticity.

\section{Up- and down-states, bursting, and background noise}

It has been suggested that being at the "edge of chaos" (Crutchfield and Young, 1990; Langton, 1990) is desirable for neural networks performing complex computational tasks (Bertschinger and Natschläger, 2004). Networks functioning in regimes that are either too chaotic or too
A

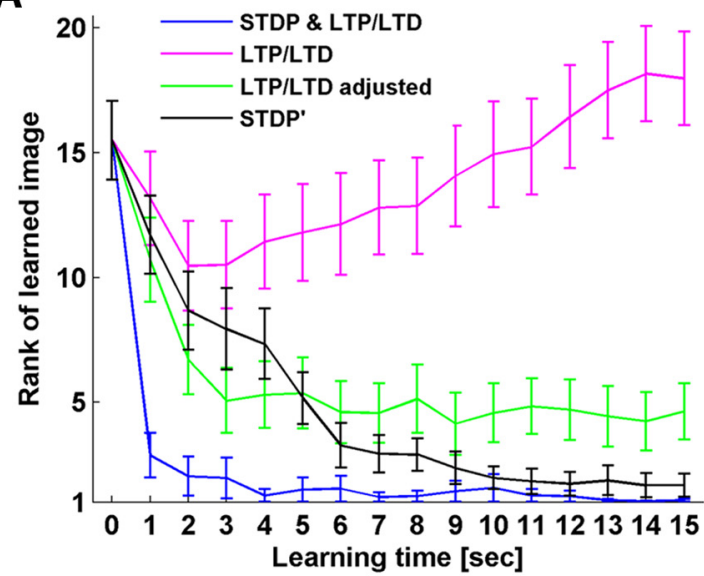

B

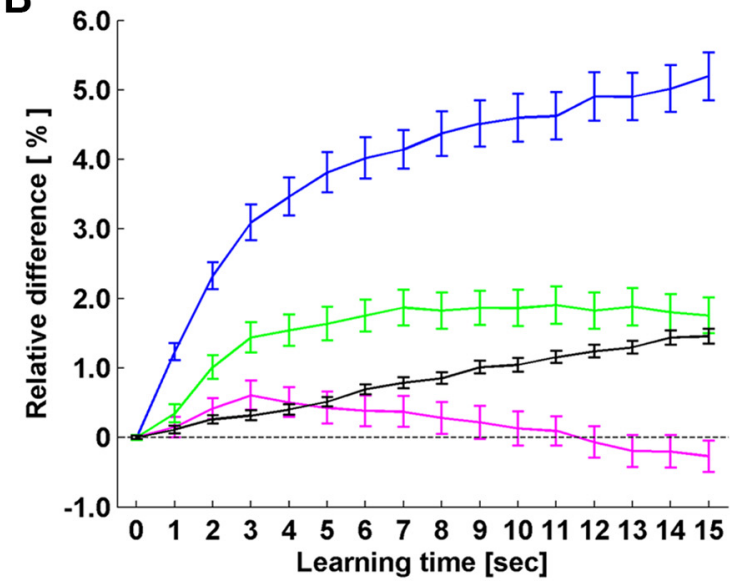

Figure 8. Impact of STDP and LTP/LTD on familiarity development. $\boldsymbol{A}$, Rank of network firing rate for the familiar image among 30 testing images is plotted as a function of learning time. The initial rank before learning is 15th on average, while an end rank of 1 st indicates the image is detected as familiar. Single face recognition simulations were repeated with full NMDAR plasticity (STDP and LTP/LTD); without STDP after removing BPAPS (LTP/LTD); without STDP but after retuning the calcium control hypothesis (LTP/LTD adjusted); and STDP plasticity alone (STDP'). Error bars represent the SEM of 30 simulations conducted on 10 networks. B, Relative difference in network firing rate to familiar and novel inputs after learning with different forms of plasticity. The relative difference is calculated as the discrepancy between network response to familiar input and the average of network responses to novel inputs, divided by the response to the familiar input after learning. Same simulations and same color code as in $\boldsymbol{A}$. 
ordered in their response to input stimuli do not perform well on computational tasks. Legenstein and Maass (2007) tested the impact of the parameters Wscale and $\lambda$ in CSIM (see Materials and Methods) for network performance on certain classification tasks. Wscale controls the strength of synaptic connections in CSIM, while $\lambda$ controls the number and average distance of synaptically connected neurons. The values of Wscale (0.9) and $\lambda(4)$ that we used in the NMDAR-LSM model were determined empirically using network performance in the familiaritynovelty tests, and they happen to be at the transitional boundary, (between ordered and chaotic responses) identified by Legenstein and Maass, an area that was reported to have a good trade-off between accuracy and generalization.

Legenstein and Maass also identified the existence of up and down-states (Cowan and Wilson, 1994) in their simulated neural network models. These states refer to dynamic regimes observed in intracellular recordings of mammalian central nervous system neurons that differ in their membrane potential and conductance properties. In the down-states, the membrane potential is hyperpolarized and stable and membrane conductance is low. In the up-states, the membrane potential is more depolarized, highly variable and membrane conductance is high, resulting from a continuous bombardment of background synaptic inputs. In neural networks, up-states are characterized by network-wide synchronized bursting activity (Johnson and Buonomano, 2007). As no bursting was observed in our simulations, it seems that the tuned Wscale and $\lambda$ parameters constrained the networks to be in down-states. To know how bursting would affect the network performance, we removed the biological boundaries of the parameters. In one simulation, we increased Wscale tenfold while preserving $\lambda$. Bursting emerged during stimulus presentations (Fig. 9A), and we measured network accuracy through single face recognition simulations and compared the results with the results described above (Fig. 9B). It turns out that in a bursting network, network performance is compromised, and more learning is required for comparable accuracy. Bursting also emerged with a twofold increase in $\lambda$ while preserving Wscale, which induced similar impairments of network accuracy.

Network accuracy is also affected by the amount of background noise current injected into the neurons (Eq. 1) during simulation. We measured network accuracy through single face recognition simulations with background noise ranging from 1 to $100 \mathrm{nA}$. Figure $9 \mathrm{C}$ shows that network accuracy is preserved until the noise exceeds $40 \mathrm{nA}$, which is unrealistically high compared with normal ranges used in CSIM. A dramatic performance decline is seen from 40 to $50 \mathrm{nA}$, and the corresponding network responses are exemplified in Figure 9D. Background noise of $50 \mathrm{nA}$ clearly introduced more random spikes during simulation. If the number of random spikes exceeds the discrepancy of firing rate that was previously used to differentiate familiarity from novelty, it is very likely to disrupt network performance.

\section{Discussion}

In the simulations described above, we have investigated the ability of recurrent spiking neural networks supplemented with NMDAR plasticity to detect familiar sensory inputs following unsupervised learning. Unsupervised learning is biologically plausible and has been shown to be critical for the development of the mammalian central nervous system, for example, in the visual pathway (Blasdel et al., 1977). In the beard versus nobeard classification simulations, our neural networks learned to differentiate "beard" and "no-beard" face images. The single face recognition simulations were conducted with a more rigorous design to investigate the specificity of familiarity detection. Networks of $20 \times 20 \times$ 5 neurons connected by NMDAR-containing synapses were able to detect a previously-seen face accurately in most of the simulations, with a pronounced and specific increase in overall firing rate for the familiar stimulus. Responses to the control groups did not deviate far from baseline.

Our simulations with larger networks (up to $50 \times 50 \times$ 6 neurons) indicated that multiple familiarity traces can be learned and stored. The storage capacity increased with available network space. Our largest network model $(15,000$ neurons) can store familiarity traces for 60 faces, before accuracy drops to chance level. One possible explanation is that within large networks, subsets of synaptic connections that are selectively potentiated or depressed for each face can co-exist, reducing the chance of a new memory overwriting an older one. This observation suggests a potential resolution for the stabilityplasticity dilemma. Considering there are billions of neurons in the human brain organized in a very large number of microcircuits, it seems reasonable, in principal, that human recognition memory has an apparently limitless capacity, as reported by Standing's experiments (1973). Nevertheless, it is important to mention that in Standing's tests, a two-alternative forced-choice paradigm was used. In a subsequent study that supported Standing's finding on memory capacity (Brady et al., 2008), the two-choice paradigm was also used. A higher rate of recall error was reported by Laeng et al. (2007), when conducting similar image recognition experiments using a yes-no paradigm with only one picture presented. Whether the two-choice paradigm is justified as a good measure for recognition and a good indication for memory capacity is arguable (Laeng et al., 2007). Our analysis relied on a comparative paradigm similar to Standing's two-alternative tests: to interpret the data and measure familiarity, a threshold needs to be set for the network firing rate, which was done empirically comparing familiar and novel responses. Once the threshold is set, the networks can also make yes-no decisions for individual inputs (Fig. 4G).

In addition to the specificity and capacity for familiarity detection, we also noticed a generalization effect in the responses of the trained networks to input stimuli. Evidence for generalization was observed when, following training, the response to novel input stimuli was increased 
A

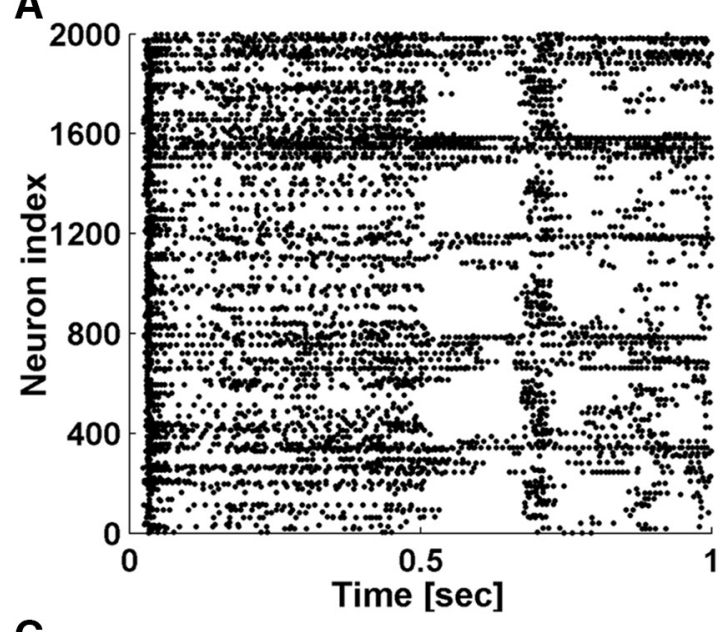

C



B

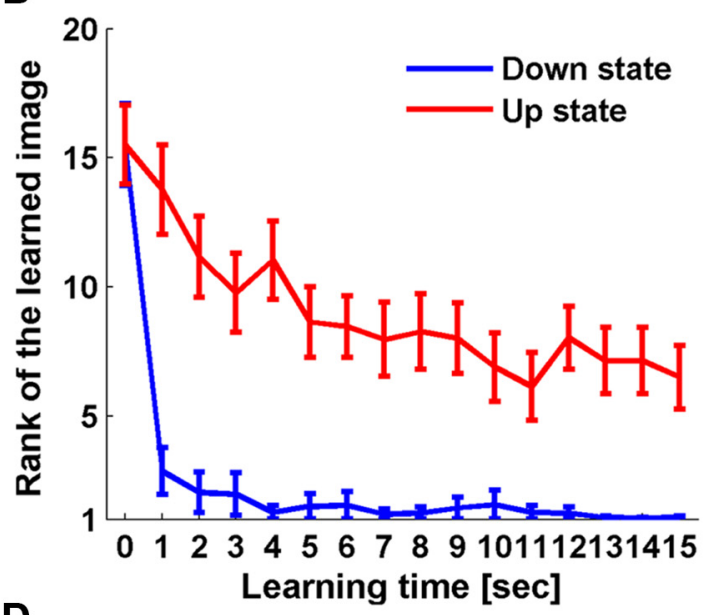

D

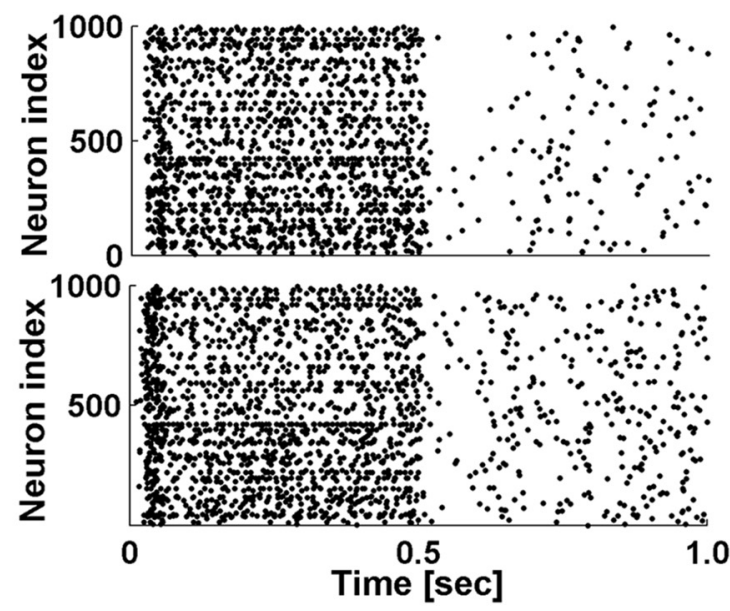

Figure 9. Effect of bursting and noise. $\boldsymbol{A}$, Response of a $20 \times 20 \times 5$ up-state network after learning a human face image for 11 s. A network burst spontaneously emerged in the interval of $0.5-1.0 \mathrm{~s}$ when no input stimulus was given. $\boldsymbol{B}$, Impact of bursting on network performance. Rank of network firing rate to the familiar image among 30 testing images is plotted as a function of learning time. Blue represents simulations with down-state networks, the same networks as we used in the single face recognition simulations. Red represents simulations with up-state networks (Wscale was set tenfold of the original value used in simulation). Error bars are the SEM of 90 simulations conducted on 30 networks. C, Impact of background noise on network performance. Average rank of network firing rate to the familiar image among 30 test images after learning for $15 \mathrm{~s}$ is plotted as a function of background noise. $\boldsymbol{D}$, Network spiking activity of a $20 \times 20 \times 5$ network to the input stimulus, simulated with $40-$ and $50-n A$ background noise, respectively.

selectively for those that belonged to the same class as the trained inputs. In the beard versus no-beard classification simulations, generalization was observed when the trained networks were able to distinguish novel beard from novel no-beard images. In single face recognition simulations, the networks generalized familiarity response to a subset of images from the same class as the presented stimulus. In the multi-face recognition simulations, the networks apparently learned the concept face after training on ten face images: they responded stronger to novel faces than their scrambled versions. Recall that for $10 \times 10 \times 5$ networks, input neurons were set to form synapses one-to-one with the first layer of the network reservoir, whereas for networks of $20 \times 20 \times 5$ or larger dimension, input neurons were set to form synapses randomly with the network reservoir by probability. Innervation by probability is a better way to use the network space and prevents overtraining of the first layer of the network reservoir. Meanwhile it disrupts the spatial patterns present in the face images, making the familiarity detection a high-dimensional challenge. Yet the generalization property remained with the randomly projected inputs, which speaks to the robustness and computational power of our networks, i.e., learning specificity was preserved while generalization was acquired. When multiple inputs from a class were learned, generalization allowed the networks to act as an unsupervised classifier, by automatically classifying input stimuli based on past sensory experience. In contrast, conventional classifiers require properly labeled data of all classes to be trained.

Fisher's discriminant analyses suggest that familiarization occurred in high-dimensional feature space. Network connections were modified and neurons in hidden circuits were recruited to respond to familiar inputs. Neurons hidden from the input layer are analogous to neurons from deep layers of a feed-forward network, or neurons from 
the downstream circuits in higher-order brain regions. Their recruitment suggests how signals can be relayed to brain regions other than the primary processing region and cause differences in neuronal activity. As the deeplayer neurons showed unique firing patterns to the familiar stimulus, their activity can be used to identify the input pattern, a function similar to the "grandmother-cells" identified by in vivo brain recordings (Quiroga et al., 2005). For decades neuroscientists have debated the possible forms of information encoding in the brain, such as parallel distributed processing versus single neuron firing. Results presented here show they are not mutually exclusive. While a familiar input is encoded in a network of thousands of neurons, it may also selectively activate single neurons in deep layers.

Familiarity studies stemmed from experiments at the behavioral level. Several groups have conducted experiments with the familiarity/novelty paradigm in vivo. Studies using fMRI measurements (Kosaka et al., 2003; Gobbini and Haxby, 2006) found an increase in the BOLD signal to familiar stimuli. Recordings in the inferior temporal lobe of behaving monkeys have demonstrated differential responses to novel and familiar images (Anderson et al., 2008). Interestingly, familiar images evoked larger-amplitude local field potentials, whereas multi-unit spiking responses were greater for novel images. Finally, a phenomenon called stimulus-selective response potentiation was identified in rodent visual cortex recordings (Frenkel et al., 2006; Cooke and Bear, 2010; Gavornik and Bear, 2014). It is a form of experience-dependent response enhancement during visual experiments. We think it supports the existence of intrinsic familiarity in the visual cortex.

A few neural network models have been proposed to study network-level learning and memory. Several of them applied STDP to various network architectures. For example, a few groups (Lazar et al., 2007; Oliveri et al., 2007; Xue et al., 2013) investigated LSMs with STDP and the results suggested an enhanced computational capability. In these models, STDP was either applied to train readout neurons or to modulate neuron excitability, rather than allowing it to directly modify synaptic weights in the network. Studies that used neural networks other than LSMs (Clopath et al., 2010; Carlson et al., 2013; Klampfl and Maass, 2013; Zheng et al., 2013; Srinivasa and Cho, 2014) have reported emerging learning and memory after applying STDP to recurrent network architectures. Nevertheless, the networks they used are either of a preimposed wiring diagram (Klampfl and Maass, 2013) or highly simplified (Srinivasa and Cho, 2014), and therefore poorly replicate cortical microcircuits. Furthermore, these studies solely consider STDP for plasticity while we combine the two major forms of plasticity, LTD/LTP (frequency rule) and STDP (timing rule), based on the calcium control hypothesis, rather than phenomenological equations. Evidence for the interplay of LTD/LTP and STDP has been found in the literature, and separating them by firing rate or spike timing might lead to an artificial dichotomy (Sjöström et al., 2001). Our simulation results suggest a mutual-enhancing effect by combining STDP with LTP/ LTD, and this could potentially explain one-shot familiarity memory (Yakovlev et al., 2008). Additionally, some of the reported models require homeostatic control (Clopath et al., 2010; Carlson et al., 2013; Zheng et al., 2013) or inhibitory-STDP (Srinivasa and Cho, 2014) to attain network stability, but in our NMDAR-LSM networks, we relied on the intrinsic balance between LTD and LTP instantiated by the calcium control hypothesis for stability.

The plasticity model we implemented was created to model bidirectional synaptic plasticity through NMDARs (Shouval et al., 2002). Whether NMDARs are solely responsible for this form of plasticity is still controversial, as voltage-dependent $\mathrm{Ca}^{2+}$ channels (Nevian and Sakmann, 2006) and metabotropic glutamate receptors (Gladding et al., 2009) have been reported to be capable of inducing synaptic plasticity as well. Yet there is growing evidence that bidirectional modifications can be induced through NMDAR-dependent pathways alone (Hunt et al., 2013; Huang et al., 2014). The results presented here demonstrate that bidirectional synaptic plasticity is sufficient to endow neural network models of generic cortical microcircuits with the ability to detect familiar sensory inputs through unsupervised learning. This has important consequences for mammalian brain development, since it suggests that these universal building blocks of the cortex have an inherent ability for familiarity detection.

In NMDAR-LSM networks, recurrent spiking neural networks expand input stimuli into high-dimensional feature space (Maass et al., 2002). Unsupervised learning altered the feature space to allow linear separation of familiar from novel faces, by formation of subnetworks specific for each input stimulus. Learning multiple inputs belonging to a class (e.g., beard, face) resulted in generalization, allowing the network to classify novel input stimuli. This relationship between familiarity detection, generalization, and classification needs to be studied in more depth.

Note Added in Proof: The title of the article was incorrectly listed in the Early Release version. The title has now been corrected.

\section{References}

Anderson B, Mruczek RE, Kawasaki K, Sheinberg D (2008) Effects of familiarity on neural activity in monkey inferior temporal lobe. Cereb Cortex 18:2540-2552. CrossRef Medline

Baudry M, Zhu G, Liu Y, Wang Y, Briz V, Bi X (2015) Multiple cellular cascades participate in long-term potentiation and in hippocampusdependent learning. Brain Res 1621:73-81. CrossRef Medline

Bertschinger N, Natschläger T (2004) Real-time computation at the edge of chaos in recurrent neural networks. Neural Comput 16: 1413-1436. CrossRef Medline

Bi GQ, Poo MM (1998) Synaptic modifications in cultured hippocampal neurons: dependence on spike timing, synaptic strength, and postsynaptic cell type. J Neurosci 18:10464-10472.

Blanke ML, VanDongen AMJ (2009) Activation mechanisms of the NMDA receptor. In: Biology of the NMDA receptor (Van Dongen AM, ed). Boca Raton: CRC Press/Taylor \& Francis.

Blasdel GG, Mitchell DE, Muir DW, Pettigrew JD (1977) A physiological and behavioural study in cats of the effect of early visual experience with contours of a single orientation. J Physiol 265: 615-636. Medline

Bliss TV, Lomo T (1973) Long-lasting potentiation of synaptic transmission in the dentate area of the anaesthetized rabbit following stimulation of the perforant path. J Physiol 232:331-356. Medline 
Brady TF, Konkle T, Alvarez GA, Oliva A (2008) Visual long-term memory has a massive storage capacity for object details. Proc Natl Acad Sci USA 105:14325-14329. CrossRef Medline

Buonomano DV, Maass W (2009) State-dependent computations: spatiotemporal processing in cortical networks. Nat Rev Neurosci 10:113-125. CrossRef Medline

Carlson KD, Richert M, Dutt N, Krichmar JL (2013) Biologically plausible models of homeostasis and STDP: stability and learning in spiking neural networks. In: The 2013 International Joint Conference on Neural Networks (IJCNN), pp 1-8.

Clopath C, Büsing L, Vasilaki E, Gerstner W (2010) Connectivity reflects coding: a model of voltage-based STDP with homeostasis. Nat Neurosci 13:344-352. CrossRef Medline

Cooke SF, Bear MF (2010) Visual experience induces long-term potentiation in the primary visual cortex. J Neurosci 30:1630416313. CrossRef Medline

Coutlee CG, Huettel SA (2012) The functional neuroanatomy of decision making: prefrontal control of thought and action. Brain Res 1428:3-12. CrossRef Medline

Cowan RL, Wilson CJ (1994) Spontaneous firing patterns and axonal projections of single corticostriatal neurons in the rat medial agranular cortex. J Neurophysiol 71:17-32. Medline

Crutchfield JP, Young K (1990) Computation at the onset of chaos. In: Entropy, complexity, and physics of information (Zurek W, ed), pp 223-269. Bouler: Addison-Wesley.

Diana RA, Yonelinas AP, Ranganath C (2007) Imaging recollection and familiarity in the medial temporal lobe: a three-component model. Trends Cogn Sci 11:379-386. CrossRef Medline

Fair DA, Cohen AL, Power JD, Dosenbach NU, Church JA, Miezin FM, Schlaggar BL, Petersen SE (2009) Functional brain networks develop from a "local to distributed" organization. PLoS Comput Biol 5:e1000381. CrossRef

Fornito A, Harrison BJ, Zalesky A, Simons JS (2012) Competitive and cooperative dynamics of large-scale brain functional networks supporting recollection. Proc Natl Acad Sci USA 109:1278812793. CrossRef Medline

Freedman DJ, Riesenhuber M, Poggio T, Miller EK (2006) Experiencedependent sharpening of visual shape selectivity in inferior temporal cortex. Cereb Cortex 16:1631-1644. CrossRef Medline

Frenkel MY, Sawtell NB, Diogo ACM, Yoon B, Neve RL, Bear MF (2006) Instructive effect of visual experience in mouse visual cortex. Neuron 51:339-349. CrossRef Medline

Froemke RC, Dan Y (2002) Spike-timing-dependent synaptic modification induced by natural spike trains. Nature 416:433-438. CrossRef Medline

Froemke RC, Debanne D, Bi GQ (2010) Temporal modulation of spike-timing-dependent plasticity. Frontiers in synaptic neuroscience 2:19.

Gale JT, Shields DC, Ishizawa Y, Eskandar EN (2014) Reward and reinforcement activity in the nucleus accumbens during learning. Front Behav Neurosci 8:114 CrossRef Medline

Gavornik JP, Bear MF (2014) Higher brain functions served by the lowly rodent primary visual cortex. Learn Mem 21:527-533. CrossRef Medline

Gerstner W, Kempter R, van Hemmen JL, Wagner H (1996) A neuronal learning rule for sub-millisecond temporal coding. Nature 383:76-81. CrossRef Medline

Gladding CM, Fitzjohn SM, Molnár E (2009) Metabotropic glutamate receptor-mediated long-term depression: molecular mechanisms. Pharmacol Rev 61:395-412. CrossRef Medline

Gobbini MI, Haxby JV (2006) Neural response to the visual familiarity of faces. Brain Res Bull 71:76-82. CrossRef Medline

Grossberg S (1980) How does a brain build a cognitive code? Psychol Rev 87:1-51. Medline

Grossberg S (2013) Adaptive resonance theory: how a brain learns to consciously attend, learn, and recognize a changing world. Neural Netw 37:1-47. CrossRef

Gupta A, Wang Y, Markram H (2000) Organizing principles for a diversity of GABAergic interneurons and synapses in the neocortex. Science 287:273-278. Medline
Gutig R, Aharonov R, Rotter S, Sompolinsky H (2003) Learning input correlations through nonlinear temporally asymmetric Hebbian plasticity. J Neurosci 23:3697-3714.

Hölscher C, Rolls ET, Xiang J (2003) Perirhinal cortex neuronal activity related to long-term familiarity memory in the macaque. Eur J Neurosci 18:2037-2046. Medline

Huang S, Rozas C, Treviño M, Contreras J, Yang S, Song L, Yoshioka T, Lee HK, Kirkwood A (2014) Associative Hebbian synaptic plasticity in primate visual cortex. J Neurosci 34:7575-7579. CrossRef Medline

Hunt DL, Puente N, Grandes P, Castillo PE (2013) Bidirectional NMDA receptor plasticity controls CA3 output and heterosynaptic metaplasticity. Nat Neurosci 16:1049-1059. CrossRef Medline

Ito M, Kano M (1982) Long-lasting depression of parallel fiberPurkinje cell transmission induced by conjunctive stimulation of parallel fibers and climbing fibers in the cerebellar cortex. Neurosci Lett 33:253-258. Medline

Johnson HA, Buonomano DV (2007) Development and plasticity of spontaneous activity and up states in cortical organotypic slices. $J$ Neurosci 27:5915-5925. CrossRef

Joshi P (2007) From memory-based decisions to decision-based movements: a model of interval discrimination followed by action selection. Neural Netw 20:298-311. CrossRef

Ju H, Xu JX, Chong E, VanDongen AM (2013) Effects of synaptic connectivity on liquid state machine performance. Neural Netw 38:39-51. CrossRef

Ju H, Dranias MR, Banumurthy G, VanDongen AM (2015) Spatiotemporal memory is an intrinsic property of networks of dissociated cortical neurons. J Neurosci 35:4040-4051. CrossRef Medline

Klampfl S, Maass W (2013) Emergence of dynamic memory traces in cortical microcircuit models through STDP. J Neurosci 33:1151511529. CrossRef

Kosaka H, Omori M, lidaka T, Murata T, Shimoyama T, Okada T, Sadato N, Yonekura Y, Wada Y (2003) Neural substrates participating in acquisition of facial familiarity: an fMRI study. Neurolmage 20:1734-1742. Medline

Laeng B, Øvervoll M, Ole Steinsvik O (2007) Remembering 1500 pictures: the right hemisphere remembers better than the left. Brain Cogn 63:136-144. CrossRef Medline

Langton CG (1990) Computation at the edge of chaos: phase transitions and emergent computation. Physica D 42:12-37. CrossRef

Lazar A, Pipa G, Triesch J (2007) Fading memory and time series prediction in recurrent networks with different forms of plasticity. Neural Netw 20:312-322. CrossRef Medline

Legenstein R, Maass W (2007) Edge of chaos and prediction of computational performance for neural circuit models. Neural Netw 20:323-334. CrossRef

Lisman J (1989) A mechanism for the Hebb and the anti-Hebb processes underlying learning and memory. Proc Natl Acad Sci USA 86:9574-9578. Medline

Liu X, Ramirez S, Pang PT, Puryear CB, Govindarajan A, Deisseroth K, Tonegawa S (2012) Optogenetic stimulation of a hippocampal engram activates fear memory recall. Nature 484:381-385. CrossRef Medline

Luscher C, Malenka RC (2012) NMDA receptor-dependent long-term potentiation and long-term depression (LTP/LTD). Cold Spring Harb Perspect Biol 4. CrossRef

Maass W, Natschläger T, Markram H (2002) Real-time computing without stable states: a new framework for neural computation based on perturbations. Neural Comput 14:2531-2560. CrossRef

Malleret G, Alarcon JM, Martel G, Takizawa S, Vronskaya S, Yin D, Chen IZ, Kandel ER, Shumyatsky GP (2010) Bidirectional regulation of hippocampal long-term synaptic plasticity and its influence on opposing forms of memory. J Neurosci 30:3813-3825. CrossRef

Markram H, Lübke J, Frotscher M, Sakmann B (1997) Regulation of synaptic efficacy by coincidence of postsynaptic APs and EPSPs. Science 275:213-215. Medline 
Massey PV, Bashir ZI (2007) Long-term depression: multiple forms and implications for brain function. Trends Neurosci 30:176-184. CrossRef Medline

Mermillod M, Bugaiska A, Bonin P (2013) The stability-plasticity dilemma: investigating the continuum from catastrophic forgetting to age-limited learning effects. Front Psychol 4:504. CrossRef Medline

Meyer T, Walker C, Cho RY, Olson CR (2014) Image familiarization sharpens response dynamics of neurons in inferotemporal cortex. Nat Neurosci 17:1388-1394. CrossRef Medline

Morgado-Bernal I (2011) Learning and memory consolidation: linking molecular and behavioral data. Neuroscience 176:12-19. CrossRef Medline

Natschläger T, Markram H, Maass W (2002) Computer models and analysis tools for neural microcircuits. In: Neuroscience databases: a practical guide (Kötter R, ed). Boston: Kluver Academic Publishers.

Nevian T, Sakmann B (2006) Spine Ca2+ signaling in spike-timingdependent plasticity. J Neurosci 26:11001-11013. CrossRef Medline

Nickerson RS (1965) Short-term memory for complex meaningful visual configurations: a demonstration of capacity. Can J Psychol 19:155-160. Medline

Oliveri A, Rizzo R, Chella A (2007) An application of spike-timingdependent plasticity to readout circuit for liquid state machine. In: Neural networks, the 2007 International Joint Conference on Neural Networks (IJCNN), pp 1441-1445.

Paradiso K, Wu LG (2009) Small voltage changes at nerve terminals travel up axons to affect action potential initiation. Nat Neurosci 12:541-543. CrossRef Medline

Pattillo JM, Artim DE, Simples JE Jr, Meriney SD (1999) Variations in onset of action potential broadening: effects on calcium current studied in chick ciliary ganglion neurones. J Physiol 514:719-728. CrossRef

Purves D, Wojtach WT, Lotto RB (2011) Understanding vision in wholly empirical terms. Proc Natl Acad Sci USA 108:1558815595. CrossRef

Quiroga RQ, Reddy L, Kreiman G, Koch C, Fried I (2005) Invariant visual representation by single neurons in the human brain. Nature 435:1102-1107. CrossRef Medline

Rapanelli M, Frick LR, Miguelez Fernández AM, Zanutto BS (2015) Dopamine bioavailability in the mPFC modulates operant learning performance in rats: an experimental study with a computational interpretation. Behav Brain Res 280:92-100. CrossRef Medline

Salter MW, Dong Y, Kalia LV, Liu XJ, Pitcher G (2009) Regulation of NMDA receptors by kinases and phosphatases. In: Biology of the NMDA receptor (Van Dongen AM, ed). Boca Raton: CRC Press/ Taylor \& Francis.
Shepard RN (1967) Recognition memory for words, sentences, and pictures. J Verbal Learning Verbal Behav 6:156-163. CrossRef

Shouval HZ, Bear MF, Cooper LN (2002) A unified model of NMDA receptor-dependent bidirectional synaptic plasticity. Proc Natl Acad Sci USA 99:10831-10836. CrossRef Medline

Sjöström PJ, Turrigiano GG, Nelson SB (2001) Rate, timing, and cooperativity jointly determine cortical synaptic plasticity. Neuron 32:1149-1164. Medline

Song S, Miller KD, Abbott LF (2000) Competitive Hebbian learning through spike-timing-dependent synaptic plasticity. Nat Neurosci 3:919-926. CrossRef Medline

Sporns O, Zwi JD (2004) The small world of the cerebral cortex. Neuroinformatics 2:145-162. CrossRef Medline

Squire LR, Wixted JT, Clark RE (2007) Recognition memory and the medial temporal lobe: a new perspective. Nat Rev Neurosci 8:872883. CrossRef Medline

Srinivasa N, Cho Y (2014) Unsupervised discrimination of patterns in spiking neural networks with excitatory and inhibitory synaptic plasticity. Front Comput Neurosci 8:159. CrossRef Medline

Standing L (1973) Learning 10,000 pictures. Q J Exp Psychol (Hove) 25:207-222. CrossRef Medline

Takeuchi T, Duszkiewicz AJ, Morris RG (2014) The synaptic plasticity and memory hypothesis: encoding, storage and persistence. Philos Trans R Soc Lond B Biol Sci 369:20130288. CrossRef Medline

Waters J, Schaefer A, Sakmann B (2005) Backpropagating action potentials in neurones: measurement, mechanisms and potential functions. Prog Biophys Mol Biol 87:145-170. CrossRef Medline

Watts DJ, Strogatz SH (1998) Collective dynamics of 'small-world' networks. Nature 393:440-442. CrossRef Medline

Wixted JT (2007) Dual-process theory and signal-detection theory of recognition memory. Psychol Rev 114:152-176. CrossRef Medline

Wu J, Rowan MJ, Anwyl R (2006) Long-term potentiation is mediated by multiple kinase cascades involving CaMKII or either PKA or $\mathrm{p} 42 / 44$ MAPK in the adult rat dentate gyrus in vitro. J Neurophysiol 95:3519-3527. CrossRef

Xue F, Hou Z, Li X (2013) Computational capability of liquid state machines with spike-timing-dependent plasticity. Neurocomputing 122:324-329. CrossRef

Yakovlev V, Amit DJ, Romani S, Hochstein S (2008) Universal memory mechanism for familiarity recognition and identification. J Neurosci 28:239-248. CrossRef Medline

Yonelinas AP (2002) The nature of recollection and familiarity: a review of 30 years of research. J Mem Lang 46:441-517. CrossRef

Zheng P, Dimitrakakis C, Triesch J (2013) Network self-organization explains the statistics and dynamics of synaptic connection strengths in cortex. PLoS Comput Biol 9:e1002848. CrossRef Medline 